

\title{
Berechnung der Druckverteilung an Flügelprofilen in gashaltiger Wasserströmung
}

\begin{abstract}
Die vorliegende Arbeit behandelt die ebene, stationäre und hompressible Strömung von gashaltigem Wasser um T'ragflügelprofile. Unter dem Begriff Gas wird dabei sowohl die im Wasser enthaltene ungelöste Luft als auch der durch auftretende Kavitation bedingte Wasserdampf verstanden. Zur Vereinfachung der Theorie ist die Eigenbewegung der Gasblasen gegenüber dem umgebenden Wasser vernachlässigt, also ein einheitliches Geschwindigkeits- und Druckfeld angenommen; außerdem wird vorausgesetzt, daß das Verhältnis von Gasmasse zu Wassermasse eine eindeutige Funktion des örtlichen Druckfeldes sei. In Anlehnung an eine in der Theorie schallnaher Gasströmungen bewährte Methode erfolgt die Berechnung des Druckfeldes in der Ümgebung der Flügelprofile aus einer nichtlinearen Integralgleichung. Die Theorie wird an einer Reilhe von Zahlenbeispielen erprobt. Es zeigt sich, daß durch den Gasgehalt des Wassers nur dann wesentliche Unterschiede gegenüber dem inkompressiblen Strömungszustand bedingt sind, wenn infolge der niedrigen örtlichen Schallgeschwindigkiet mit Verdichtungsstößen abgeschlossene lokale Überschallgebiete auftreten. Vergleiche mit aus der Literatur entnommenen Messungen geben eine zumindest qualitative Übereinstimmung mit den theoretischen Ergebnissen.
\end{abstract}

The paper is concerned with the two-dimensional steady and compressible flow of water with gas-content around hydrofoils. Gas means as well the content on free air in the water as the vapour occurring from cavitation. For the sake of simplicity we have neglected the movement of gas-bubbles relative to the surrounding water. Therefore only one velocity - and pressurefield is adopted. Besides we have assumed, that the mass-ratio of gas to water is only a function of the local pressure. Following an efficient method of the theory of transsonic gasdynamics, the pressure-field around the profil is calculated solving a non-linear integralequation. The theory is tested on some numerical examples. It turns out, that the gas-content in water gives only then remarkable differences in comparison with the incompressible pressure-field, if because of the low velocity of sound local supersonic regions occur, which end with shocks. $A$ comparison with available experimental data shows at least a qualitative agreement with the theoretical results.

В иастоящей работе рассматривается плоское, стационарное, снимаемое обтекание профилей крыла водой, содержащей газ. При этом подразумеваетсл под газом как и находящийся в воде нерастворений воздух так и водяннй пар, возникающий вследствие наличия ғавитации. Для упрощения тсории собственне движение пузырей газа относительно окружающей воды пренебрегается. Следовательно принимается наличие однородного поля слоростей и давлений. Кроме этого предполагается, что отношение массы газа $k$ массе воды является однозначной функцией местного поля давления. Примыная к испытанному в теории околозвуковых потоков методу, расчёт поля давления вблизи профилей крыла проиэводится с помопью нелинейного интегрального уравнения. Теория проверяется рядом числових примеров. Оказывается, что обусловленные наличием газа в воде сушественные разницы по отношепию $k$ несжимаемому потоку наблюдаются только тогда, когда вследствие низкой локальной скорости звука возникают ограниченные скачками уплотнения местные области сверхзвуговых скоростей. Взятые из литературы для сравнения результаты замеров согласовываются с теоретическими результатами по крайней мере качественно.

\section{Einleitung und Übersicht}

Die vorliegende Untersuchung befaßt sich mit der ebenen stationären Strömung um Tragflügelprofile in gashaltigem Wasser. Unter letzterem wollen wir zusammenfassend sowohl Wasser mit einem Gehalt an ungelöster Luft verstehen als auch Wasser mit Blasen, welche auf'Dampfkavitation beruhen.

Es ist bekannt, daß schon durch einen geringen Volumenanteil an ungelöster Luft oder auch an Dampf die Kompressibilität des Wassers erheblich zunimmt. Die örtliche Schallgeschwindigkeit kann dabei weit (d. h. durchaus zwei Größenordnungen bei technisch wichtigen Strömungen) unter den für reines Wasser geltenden Wert von etwa 1500 bis $1400 \mathrm{~m} / \mathrm{sec}$ absinken [1], [2], [3], [4].

Das Ziel der hier vorgelegten Arbeit ist es zu untersuchen, inwieweit das Druckfeld in der Umgebung eines 'I'ragflügelprofils auf Grund der erhöhten Kompressibilität gashaltigen Wassers gegenüber den Verhältnissen in reinem Wasser modifiziert wird. Um mit einem vertretbaren Aufwand zu numerischen Ergeb. nissen zu kommen, beschränken wir uns auf die ebene stationäre und reibungsfreie Strömung.

Eine wichtige Größe für unser Problem ist das örtliche Verhältnis von Gasmasse $M_{G}$ zu Wassermasse $M_{W}$ in der Strömung; wir bezeichnen es mit $\mu=M_{G} / M_{W}$.

Genau genommen ist dieses Massenverhältnis $\mu$ eine orts- und zeitabhängige Funktion, welche durch eine ganze Anzahl physikalischer Effekte beeinflußt wird. Zum einen charakterisiert $\mu$ die im Wasser enthaltene ungelöste Luftmasse. Letztere ändert sich, wenn das Gleichgewicht zwischen gelöster und ungelöster Luft infolge Variation des lokalen Druckfeldes gestört wird; auch die Eigenbewegung der Luftblasen relativ zum umgebenden Wasser wird dabei eine Rolle spielen. Zum anderen sind Luftblasen in ihrer Eigenschaft als Kavitationskeime auch für die in den Stellen niedrigen Druckes wesentliche Dampfkavitation bedeutsam. In denjenigen Gebieten des Strömungsfeldes, in denen Dampfkavitation herrscht, wird das Massenverhältnis $\mu$ vor allem durch die Verdampfungsvorgänge und die Blasendynamik einschließlich der Wechselwirkung zwischen den Blasen bestimmt sein. Der freie Luftgehalt ist dann von geringerem Gewicht.

Aus diesen kurzen Úberlegungen wird bereits deutlich, wie schwierig eine genaue, den physikalischen Gegebenheiten entsprechende Berechnung von $\mu$ sein würde. 
Bei viner Unterscheidung zwischen luft und Wasserdampf hätte man unter Beräcksichtigung der Eigenbewegung der Blasen eine echte Drei-Phasen-Strömung mit drei verschiedenen untereinander in Wechselwirkung st ehenden Geschwindigkeitsfeldern. Überdenkt man jedoch die mathematischen Konsequenzen, so crgibt sich, daß eine solche 'Theorie nach dem derzeitigen Stand der Forschung kaum sicher formulierbar und schon gar nicht mit einem vertretbaren Aufwand numerisch auswertbar wäre. Man muB sich vielmehr mit einem wesentlich vereinfachten Modell begnügen, wie dieses in der vorliegenden Arbeit auch geschieht.

J)azu geliört zunächst die Vernachlässigung der Eigenbewegung von l.uft. und Kavitationsblasen gegenïber clem umgebenden Wasser. Dadurch haben wir es nur noch mit cinem einzigen Geschwindigkeitsfeld zu tun, das wir außerhalb etwa auftretender Verdichtmosstöße (oder wie man auch sagt Kavitationsstöße) als Potentialströmung behandeln werden. Jor Einflul3 der Zähigkeit des Mediums wird ohnehin nicht berücksichtigrt.

Weiter wollen wir annclımen, daß3 das örtliche Massenverhältnis / eine eindeutige Funktion des örtliehen 1) ruckfeldes $p$ sei und gleichzeitig die ungelöste luftmasse und die Dampfmasse beschreibt. Thermodynamiscb entspricht dies der Vornussetzung eines Gleichgewichtszustandes für das Strömungsmedium. Da $\mu$ auf jeden Fall mit almohmendem Druck wachsen mul, Jiegt ein Ansatz der Form

$$
\mu=\mu_{w}\left(\frac{p_{w}}{p}\right)^{2} \quad(i . \geqq 0)
$$

nahe mit einem zunächst freien lixponenten $\%$. Die Größ3en $p_{\infty}, \mu_{\infty}$ bezeichnen dabei einen Ausgangs\%ustand, ж. B. die Verhält nisse weit ror dem betrachteten Flügelprofil. Der Sonderfall $\lambda=0$ entspricht einem konstanten Massenverhältnis. Der Ansatz (1) bat sich rom theoretischen Standpunkt aus bei der J3ehandlung der strö. mungsmechanischen Gleichungen (Kontinuitätsgleichung, Impulssatz, linergiesatz, Stoßrelationen) als recht

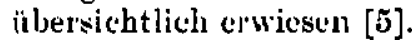

\section{$\because$ Dic st rïmungsmechanischen tileichmmgen}

Im Rahmen der hicr beabsichtigten Untersuchung dess stationären Druckfeldes in clet Umgebung eines l'ragflügelprofils ist es ohne Bingehen auf die akustische Abstrahlung ohne weiteres zalässig, das reine Wasser als inkompressibel, also

$$
e_{w}=\text { konst. }
$$

anzunchmen. Die Kompressibilität ist dann lediglich durch die Gasdichte $\varrho_{0}$ bedingt. Unter $\varrho_{G}$ rerstehen wir zusammenfassend sowohl die Luftdichte als auch die Dampfdichte.

Wir bezeichnen mit $\alpha$ das Verhältnis von Gasvolumen $V_{g} z u$ Wasservolumen $V_{w}$ jn einem kleinen Vo. lumenbereich $\gamma_{\theta}+V_{W}$ des Strömungsfeldes, also

$$
\alpha=\frac{V_{G}}{V_{W}}=\mu \frac{Q_{W}}{Q_{G}} \text {. }
$$

l)amit wirl die (ientiscludichte

$$
\omega=\omega_{w} \frac{1+\mu}{1+\alpha}
$$

Für den Gasanteil der Strömung setzen wir die Gültigkedit cler Zustandscrlejełung

$$
p=\varrho_{G} T R \quad(R=\text { Gaskonstante })
$$

voraus. Unter der einleuchtenden Annahme, daß die Gasblasen die glejehe 'lemperatur $T$ ' wie das umgebende Wasser haben und diese Wassertemperatur überhaupt konstant bleibt (isotherme Zustandsänderung), folgt weiter $\left.{ }^{1}\right)$

$$
\frac{p \alpha}{\mu Q_{W}}=\text { konst. }
$$

uder auch mit (1) und (2):

$$
\alpha=\alpha_{\infty}\left(\frac{p_{\infty}}{p}\right)^{1+\lambda}
$$

1)er Index ", $\infty$ bezeichnet Bezugswerte, clie sich auf den Zustand weit vor dem lilügelprofil, also auf die Anntrömung beziehen.

Für die Anwenclung in der Propellerhydrodynamik haben vor allem Strömungen mit einem relativ kleinen Luft volumengehalt Bedeutung. Wir wollen daher $\alpha<1$ voraussetzen, und da auBerlem $Q_{G} / \Omega_{w} \ll 1$ ist, dürfen wir davoul ausgelien, daß etwa $\mu<10^{-3}$ bleibt. Dann läßt sjoh überall

$$
1+\mu \approx 1
$$

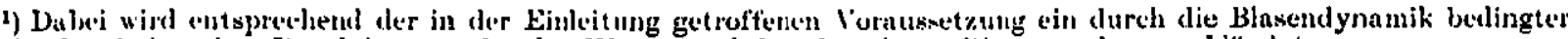
Conterschied wwiselien clom Druck im umgebenden Wasser und dem Druck am 13lusenrund vernachlässigt. 
setzen und mit dieser Vercinfachung folgt aus (4) und (7) für die Gemischdichto

$$
\varrho=\Omega_{w}\left[1+\alpha_{\infty}\left(\frac{p_{\infty}}{p}\right)^{1+\lambda}\right]^{-1} \text {. }
$$

Aus (9) (rhalten wir für die Schallgeschwindigkeit $c$ des gashaltigen Wassers die Relation

$$
c^{2}=\frac{p_{\infty}}{\varrho_{W}}\left(\frac{p_{\infty}}{p}\right)^{\lambda}\left[\frac{\alpha_{\infty}+\left(\frac{p}{p_{\infty}}\right)^{1+\lambda}}{\alpha_{\infty}(1+\lambda)}\right]^{2}=\frac{p_{\infty}}{\varrho_{W}}\left(\frac{\alpha_{\infty}}{\alpha}\right)^{\frac{1}{1+\lambda}} \frac{[1+\alpha]^{2}}{\alpha(1+\lambda)} .
$$

Formel (10) zeigt, daB die Schallgeschwindigkeit stark rom örtlichen Druckfeld abhängt und in Bereichen niedrigen Druekes schr kleine Werte annehmen kann. Wegen der vorausgesetzten Inkompressibilität des reinen Wassers gemäß (2) darf in Gleichung (10) der Grenzübergang zu reinem Wasser mit $\alpha_{\infty} \rightarrow 0$ nicht vor. genommen werden. Jedloch ist es ohne weiteres möglich [5]. die Darstellung (10) entsprechend zu verallgemeinern, für die Behandlung des vorliegenden Problems ist dieses aber nicht notwendig.

Im Rahmen der Vereinfachung (S) ergibt sich für die innere Fnergie e der Strömung

$$
e=\varepsilon_{W} T=\frac{\varepsilon_{W}}{R} \frac{\alpha p}{\mu Q_{W}}=\frac{1}{0,07} \frac{p}{\mu}\left[\frac{1}{\varrho}-\frac{1}{\varrho_{W}}\right]
$$

mit $\varepsilon_{W}$ als der spezifisch' $\cdot$ Wärme des Wassers. $R / \varepsilon_{W}=0,07$ (für Luft). Aus Gleichung (11) folgt mit $c^{2}=\left(\frac{p}{\varrho^{2}}-\frac{\hat{\sigma} e}{\partial \varrho}\right)\left(\frac{\partial e}{\partial p}\right)^{-1}$ wieder das Ergebnis (10), wic man leicht bestätigt.

Das Geschwindigkeitsfeld $v$ in der Umgebung des betrachteten Flïgelprofils setzen wir (abgesehen rom Bereich etwa auftretender Verdichtungsstōße) als Potentialströmung in der' Form

$$
v=u_{\infty}\left(e_{x}+\delta_{0} e_{y}\right)+\operatorname{grad} \phi
$$

an. Dabei ist $u_{\infty}\left(e_{x}+\delta_{0} e_{y}\right)$ mit $\delta_{0}^{2} \ll 1$ die Anströmung weit vor dom Profil. Wie in der Theorie schlanker Profile üblich, legen wir die (abgesehen von der unmittclbaren Umgebung rles Staupunktes) in guter Näherung erfüllte Voraussetzung

$$
(\text { grad } \Phi)^{2} \ll u_{\infty}^{2}
$$

zugrunde. Damit nehmen die Erhaltungssätze von Impuls (Wiclerssche Gleichung) und Masse (Kontinuitäts. gleichung) die Form an:

$$
u_{\infty} \frac{\partial \Phi}{\partial x}+\int \frac{d p}{\varrho(p)}=\text { konst. , } \quad \frac{\partial^{2} \Phi}{\partial x^{2}}+\frac{\partial^{2} \Phi}{\partial y^{2}}=\frac{u_{\infty}^{2}}{c^{2}} \frac{\partial^{2} \Phi}{\partial x^{2}} .
$$

Das in der ersten Glejehung auftretende Integral läßt sich mit (9) unmittelbar auswerten; für $c^{2}$ ist der Wert aus Formel (10) einzusetzen. Fs ergibt sich

$$
\mathrm{J}-\frac{\varrho_{W} u_{\infty}}{p_{\infty}} \frac{\partial \Phi}{\partial x}=\frac{p}{p_{\infty}}-\frac{\alpha_{\infty}}{\lambda}\left[\left(\frac{p_{\infty}}{p}\right)^{\lambda}-1\right]
$$

und

$$
\frac{\hat{\partial}^{2} \Phi}{\partial x^{2}}+\frac{\partial^{2} \Phi}{\partial y^{2}}=\alpha_{\infty} \frac{\rho_{H} u_{\infty}^{2}}{p_{\infty}}(1+\lambda)\left(\frac{p}{p_{\infty}}\right)^{\lambda}\left[\left(\frac{p}{p_{\infty}}\right)^{\lambda+1}+\alpha_{\infty}\right]^{-2} \frac{\partial^{2} \Phi}{\partial x^{2}}
$$

Die beiden Relationen (14) und (15) stellen ein nichtlineares J)ifferentialylcichungssystem zur Berechnung des Geschwindigkeitspotentials $\Phi$ und des Druckes $p$ dar. Sie gelten aber nur in den stoßfreien Bereichen des Strömungsfeldes, wo sich alle Funktionen stetig differenzierbar rerhalten.

Auf der Saugseite eines Flügelprofils können wegen der dort herrschenden niedrigen örtlichen Schall. geschwindigkeit loknle Bereiche mit Úberschallzustand auftreten, die mit einem Verdichtungsstoß abgeschlossen werden. Wir müssen daher für die vollständige Berchhnung der Strömung außer Gleichung (14) und (15) noch die Stoßrelationen berücksichtigen.

Wir bezeichnen mit $U_{n}$ die Geschwindigkeitskomponente senkrecht und mit $U_{t}$ die Komponente parallel z.u der als Gerale angenommenen Stoßlinie und kennzeichnen alle Größen ror dem Verdichtungsstoß mit $\sim$, dahinter mit $\wedge$.

Die Erhaltungssätze von Masse, Impuls und Fnergie liefern die Jeeziehungen

$$
\begin{aligned}
& \tilde{U}_{\imath}=\hat{U}_{\imath} ; \quad \frac{\tilde{U}}{1+\tilde{a}}=\frac{\hat{U}}{1+\hat{\alpha}} ; \quad \frac{\tilde{p}}{Q_{W}}+\frac{\widetilde{U}_{n}^{2}}{1+\tilde{\alpha}}=\frac{\hat{p}}{Q_{W}}+\frac{\hat{U}_{n}^{2}}{1+\hat{\alpha}} ; \\
& \frac{1}{2} \tilde{U}_{n}^{2}+\frac{\tilde{p}}{Q_{W}}\left(1+\tilde{\alpha}+\frac{\tilde{a}}{0,07 \tilde{\mu}}\right)=\frac{1}{2} \hat{U}_{n}^{2}+\frac{\hat{p}}{Q_{W}}\left(1+\hat{\alpha}+\frac{\hat{\alpha}}{0,07 \hat{\mu}}\right) .
\end{aligned}
$$


Durch eine elementare Rechnung folgrt aus (16) unter Berücksichtigung von (10) zunächst:

$$
\frac{\hat{p}}{\tilde{p}}=1+\frac{1-\hat{\alpha} / \tilde{\alpha}}{1+\lambda}\left(\frac{\widetilde{U}_{n}}{\tilde{c}}\right)^{2} ; \quad \frac{\hat{o}}{\tilde{\omega}}=\frac{\widetilde{U}_{n}}{\hat{U}_{n}}=\frac{1+\tilde{\alpha}}{1+\hat{\alpha}} .
$$

Wir setzen weiter voraus, daß die Abhängigkeit (1) auch über den Stoß hinweg gültig bleibt und beachten die Näherung (8). Dann liefert eine elementare Rechnung nach Elimination von $\tilde{p}, \hat{p}, \hat{U}_{n}$ die noch fehlende Be. stimmungsgleichung für $\hat{\alpha} / \tilde{\alpha}$, nämlich:

$$
\frac{\hat{\alpha}}{\tilde{a}}\left(1+\frac{1-\hat{\alpha} / \tilde{a}}{1+\hat{\imath}}\left(\frac{\tilde{U}_{n}}{\tilde{c}}\right)^{2}\right)^{\hat{+}+1}=1 .
$$

Gleichung (18) enthält natürlich stets die triviale Iösung $\hat{\alpha}=\tilde{\alpha}$. Die dem Verdichtungsstoß entsprechenden Werte sind $z$. B. für $i=0$ :

und für $\rangle=1$ :

$$
\tilde{\alpha} / \hat{\alpha}=\tilde{U}_{n}^{2} / \tilde{c}^{2} \equiv \widetilde{U}_{n}^{2} \text {, }
$$

$$
\tilde{\alpha} / \hat{\alpha}=\tilde{U}_{n}^{2}\left[\frac{1}{2}+\frac{1}{8} \widetilde{H}_{n}^{2}+\frac{1}{2} \widetilde{I}_{n} \sqrt{\frac{1}{2}+\frac{1}{16} \tilde{U}_{n}^{2}}\right] .
$$

Mit $\hat{\alpha} / \tilde{\alpha}$ liegen gemäß Gleichung (17) auch die übrigen Größen des Strömungszustands hinter dem Stoß fest. Sie lassen sich eindeutig aus dem Strömungsfeld vor dem Stoß berechnen.

Bei der hier untersuchten Strömung um schlanke Tragflügelprofile kann man in guter Näherung annehmen, daß die Stoßlinie senkrecht zur l'rofilsehne, also zur $x$-Achse (vgl. Bilder 1 und 2), verläuft. Dann ist $U_{t}^{2} \ll U_{n}^{2}$ und $\widetilde{Y}_{n}=\widetilde{U}_{n} / \tilde{c}$ stellt die örtliche It ACHzahl vor dem StoB dar.

\section{Berechnung Hes I)ruckfeldes am Tragflitgelprofil}

Um das Druckfeld in der Umgebung des Flügelprofils zu bestimmen, haben wir das System der Differentialgleichungen (14), (15) zu lösen und zwar sowohl unter Erfüllung der üblichen Strōmungsrandbedingung am Profil als auch unter Beachtung der Stoßrelationen (17), (1S) längs einer sich auf der Saugseite etwa bei $x=x_{1}$ von $y=0$ bis $y=l_{1}$ erstreckenden Stoßlinie (vgl. Bilder 1 und $\stackrel{\eta}{2}$ ). Is ist zweckmäßig, eine verallgemeinerte Druckfunktion $\mathfrak{o}$ cinzuf ühren, durch die Definition

$$
\jmath= \begin{cases}p / p_{\infty}+\frac{\alpha_{\infty}}{\lambda}\left(1-\left(\frac{p_{\infty}}{p}\right)^{\lambda}\right), & (\hat{\lambda} \neq 0) \\ p / p_{\infty}+\alpha_{\infty} \ln \frac{p}{p_{\infty}} ; & (\lambda=0) .\end{cases}
$$

Dann nimmt Gleichung (14) die Gestalt an:

$$
\mathfrak{\jmath}=1-\frac{\varrho_{W} u_{\infty}}{p_{\infty}} \frac{\partial \Phi}{\partial x}
$$

Wie eine elementare Rechnung zeigt, läßt sich die Differentialgleichung (15) in die Form bringen

$$
\Delta \Phi=u_{\infty} \frac{\partial}{\partial x} \ln \left[1+\alpha_{\infty}\left(\frac{p_{\infty}}{p}\right)^{1+\lambda}\right] .
$$

Im Zusammenhang mit den Gleichungen (19) und (20) vermerken wir noch: Im Fall der inkompressiblen Strömung ohne Gasgehalt, also für $\alpha_{\infty}=0$, kann das Druckfeld bei genügend großen Werten ron $u_{\infty}(\partial \Phi / \partial x)$ formal negativ werden; die Strömung wird dann also nicht mehr realistisch erfaßt. Bei Berücksichtigung eines

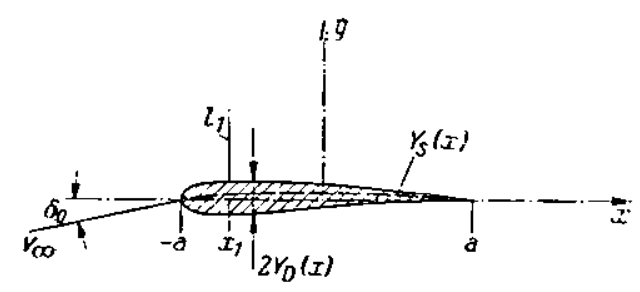

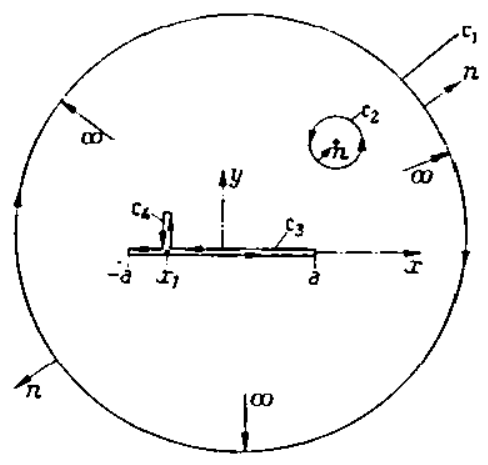

1;ild 2. Integrationshereich und Integrationswege 
Gasgelalts mit $a_{\infty} \neq 0$ wircl nur noch die in diesem Fall nicht mit $p / p_{\infty}$ äbereinstinmende Druckfunktion 5 (welche keine physikalische Bedeutung hat) negativ; der Druck $p$ sellsst blejut stets positiv. Denl Grenzwert $p \rightarrow 0$ entspricht $\} \rightarrow-\infty$ wie ein Blick auf Gleichung (19) zeigt.

Für die Iösung des nichtlincaren Differentialglejchung (21) mit den zusätzlichen kelationen (19), (20) und den Stoßbeziehtugen (17), (18) verwonden wir eine Integralglejehungsmethode, die ron der Gkswsischen Formel der l'otentialtheoric ausgeht. Ein ähnliches Vorgehen hat sich bereits in der Theorie cler schallnahen Gas-Strömungen mit Iokalen 'Jberschallgebieten bewälnt [6]. Dic Gruexsche Forme]

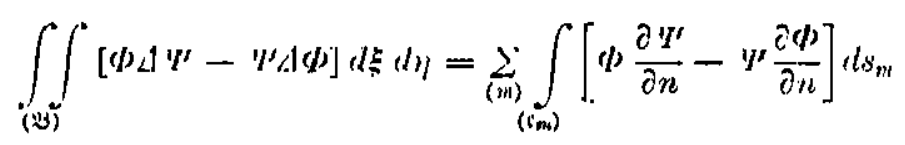

mit

$$
y^{\prime}=\ln 1^{\prime}(x-\xi)^{2}+(y-\eta)^{2}=\ln r
$$

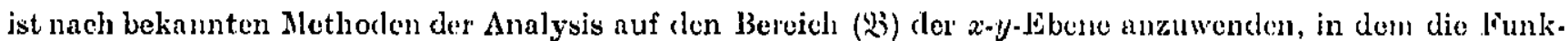
tionen $\Phi$ und $\Psi^{\prime}$ zwoimal stetig differonzicrbar sind. Tetzteres ist für $\Phi$ längs der Profilsolne im 33 areich $-\alpha \leqq x \leqq a$ der $x$-Achso und an der StoBlinie $x=x_{1}, 0 \leqq y \leqq l_{1}$ ticht der Full. Die Berandung des Bereichs (23) besteht daher außer a us den Kreiseu $\left(c_{1}\right)$ mit sehr großem und $\left(c_{2}\right)$ um den Aufpunkt $(x, y)$ mit infinitesimalen Radius noch aus zwei weiteren Anteilen: Die Randkurve $\left(c_{3}\right)$ schließt die l'rofilsehne und dic Randkurve $\left(c_{4}\right)$ die StoBlinie aus dem Bereich (B) aus. (vgl. Bild 2).

In Gleichung (22) ist $n$ dio äußere Normale, und wir verabreden, daß die Ränder für die Auswertung der Iinienintegrale so durchlaufen worden, daß das Gebict (ソ) zur Rechten liegt.

Wie üblich setzen wir voraus, claß $/$ sich im Unendlichen so verhält, dab

$$
\int_{\left(c_{2}\right)}=\lim _{r \rightarrow \infty} \int_{0}^{2 \pi}\left[\phi \frac{1}{r}-\frac{\partial(t)}{\partial r} \ln r\right] r d r_{1}=0
$$

angenommen werden kann. Weiter folgt mit, $u=-r$ :

$$
\int_{\left(r_{2}\right)}=\lim _{r \rightarrow 0} \int_{0}^{2 \pi}\left[-\phi \frac{1}{r}+\frac{\partial \phi}{\partial r} \ln r\right] r d r_{2}=-2 \pi \Phi(x, y) .
$$

Wir schreiben nun die Diskontinuitäten der Funktion $\Phi$ uncl ilırer Normalableit ungen, weiche läng's der Linien $\left(c_{3}\right)$ und $\left(c_{4}\right)$ anftreten, ju der Abkürzung

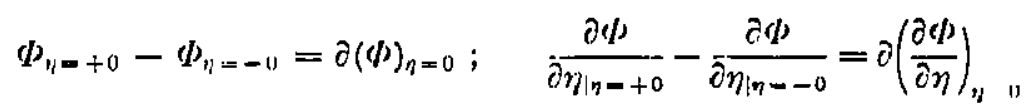

$$
\begin{aligned}
& \not D_{\xi-x_{1}+0}-\Phi_{\xi-x_{1}} 0=\partial(d)_{\xi=x_{1}} ; \quad \frac{\partial \Phi}{\partial \xi_{\mid \xi=x_{1}+0}}-\frac{\partial \phi}{\partial \xi_{\mid \xi-x_{3}-0}}=\partial\left(\frac{\partial \Phi}{\partial \xi}\right)_{\xi=x_{1}}
\end{aligned}
$$

und erlanlten no für dio boiden restlichen Randintegralo:

$$
\begin{aligned}
& \int_{\left(\xi_{3}\right)}=\int_{-a}^{a}\left[-\partial(\Phi) \frac{\partial \ln r}{\partial \eta}+\partial\left(\frac{\partial W}{\partial \eta}\right) \ln r\right]_{\eta=0} d \xi ; \\
& \int_{\left(r_{0}\right)}^{\infty}=\int_{0}^{l_{1}}\left[-\partial(\Phi) \frac{\partial \ln r}{\partial \xi}+\partial\left(\frac{\partial d}{\partial \xi}\right) \ln r\right]_{\xi=x_{1}} d \eta .
\end{aligned}
$$

In (Q⿱⺈) ist $\Delta \Psi=0$, und $\Delta \Psi$ durch die zu lösende Differentialgleichung (21) bestimmt. Unter Berücksichtigung von (23) bis (27) nimmt die Grewssche liolmel (22) als Lösung von (21) dio (iestalt an:

$$
\begin{aligned}
& 2 \pi(l)=\int_{-a}^{a}\left[\partial\left(\frac{\partial(\phi)}{\partial \eta}\right) \ln r-\hat{\imath}(\Phi) \frac{\partial \ln r}{\partial \eta}\right]_{\eta=0} d \xi+\int_{0}^{l}\left[\partial\left(\frac{\partial \phi}{\partial \xi}\right) \ln r-\lambda(\phi) \frac{\partial \ln r}{\partial \xi}\right]_{\xi=x_{0}} d \eta+\psi \\
& +\iint_{(\mathbb{S})} u_{\infty} \frac{\partial}{\partial \xi} \ln \left[1+\alpha_{i, \infty}\left(\frac{p_{\infty}}{p}\right)^{1+\lambda}\right] \ln r d \xi d \eta
\end{aligned}
$$


Für clie Bercchnung cles Druckfeldes und die Frfüllung der Strömungsrandbedingung am lị̆ügel benötigen wir die Ableitungen $\partial \Phi / \partial x, \partial \Phi / \partial y$ des Potentials (28). Dicso lassen sich mit Hilfe der Integralformeln

$$
\begin{aligned}
& \int_{-a}^{a} \partial(\Phi) \frac{\partial^{2} \ln r}{\partial x \partial \eta} d \xi=\int_{-a}^{a} \partial\left(\frac{\partial \Phi}{\partial \xi}\right) \frac{\partial \ln r}{\partial \eta} d \xi, \quad \int_{-a}^{a} \partial(\Phi) \frac{\partial^{2} \ln r}{\partial y \partial \eta} d \xi=-\int_{-a}^{a} \partial\left(\frac{\partial \Phi}{\partial \xi}\right) \frac{\partial \ln r}{\partial \xi} d \xi \\
& \int_{0}^{t_{1}} \partial(\Phi) \frac{\partial^{2} \ln r}{\partial x \partial \xi} d \eta=0 ; \quad \int_{i}^{b} \partial(\phi) \frac{\partial^{2} \ln r}{\partial y \partial \xi} d \eta=0 .
\end{aligned}
$$

in eine übersichtlichere form bringen. $\left.{ }^{2}\right)$ Dafïr ersetzen wir gemäls (ileichung (20) aulßerdem noch $\partial \Phi / \partial x$ durch 6); cs crgibt sich:

$$
\begin{aligned}
& \zeta=1+\frac{1}{2 \pi} \int_{-a}^{a} \partial(\delta)_{i=0} \frac{y d \xi}{\left(x-\frac{\partial \xi}{\xi)^{2}}+y^{2}\right.}-\frac{1}{2 \pi} \frac{\varrho_{w} u_{\infty}}{p_{\infty}} \int_{-a}^{a} \partial\left(\frac{\partial \phi}{\partial \eta}\right)_{\eta=0} \begin{array}{c}
(x-\xi) d \xi \\
(x-\xi)^{2}+y^{2}+
\end{array}
\end{aligned}
$$

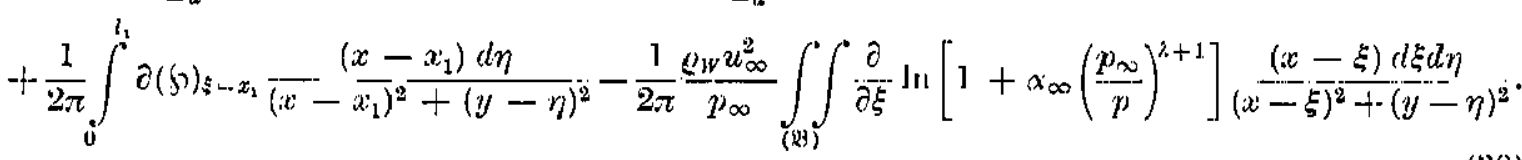

$$
\begin{aligned}
& \frac{1}{u_{\infty}} \frac{\partial \phi}{\partial y}=\frac{\mathrm{L}}{2 \pi} \frac{p_{\infty}}{Q_{w} u_{\infty}^{2}} \int_{-u}^{a} \partial(\zeta)_{y=0} \frac{(x-\xi) d \xi}{(x-\xi)^{2}+y^{2}}+\frac{1}{2 \pi} \int_{-a}^{u} \frac{1}{u_{\infty}} \partial\left(\frac{\partial \phi}{\partial \eta}\right)_{\eta=0} \frac{y d \xi}{\left(x^{2}-\xi\right)^{2}+y^{2}}-
\end{aligned}
$$

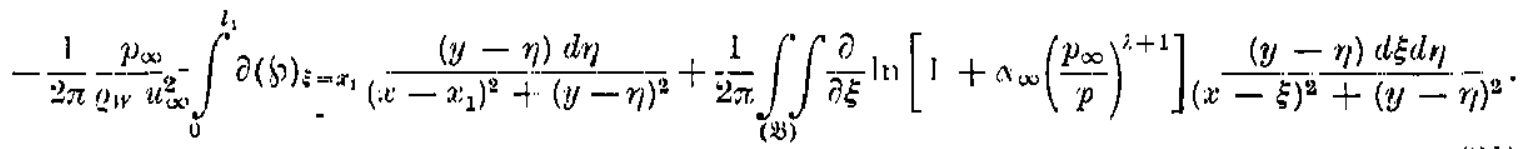

ISine Botrachtung der Gleichungen (30) und (31) zeigt, daß sie in Form der jeweils etsten beiden Integrale über den Bereich $-a \leqq \xi \leqq a$ der J'rofilsehne genau die Terme enthalten, welcho auch bei reinem Wasser ohne Gasgehalt vordanden wären. Man braucht nur zu bedenken, daB

$$
\begin{aligned}
& \partial(\xi)_{y=0}=-\frac{\varrho_{w} u_{\infty}}{p_{\infty}} \partial\left(\frac{\partial \phi}{\partial x}\right)=-\frac{\varrho_{w} u_{\infty}}{p_{\infty}} \gamma(x), \\
& \partial\left(\frac{\partial \phi}{\partial y}\right)_{y=0}=\xi^{\prime} u_{\infty} Y_{\nu}^{\prime}(x),
\end{aligned}
$$

ist mit $\gamma(x)$ als Wirbeldichte und $Y_{\nu}(x)$ als Profildiekendinie. Dio weiteren 'lorme in Gleichung (30) und (31) sind durch die Komprossibilität des gashaltigen Wassers bedingt. J)ic Joppelintegrale über (23) geben den Einflu 3 der nichtlinearen rechten Seite von Gleichung (21) wieder und treten auch dann auf, wenn das Strömungsfeld überall Unterschallzustand bchält. Die beiden Integrale über cine bei $x=x_{1}$ im 1 ereich $0 \leqq y \leqq l_{1}$ verlaufende Stoßlinie sind natürlich nur dann vorhanden, wenn ein lokales mit einom Verdichtungsstoß abgeschlossenes Üborschallgebiet auf der P'rofilsaugseite vorliogt. Aus (30) und (31) er'gibt sich dic interessante 'Tatsache, daß sich def Iinfluß eines Verdichtungsstoßes auf das Strömungsfeld mathematisch als Senkenverteilung der Stärlie

$$
q_{\text {stud }}=-u_{\infty} \partial(\delta)_{x-x_{+}} \frac{p_{\infty}}{\varrho_{W} u_{\infty}^{2}} \cdot \cdot=\partial\left(\frac{\partial \phi}{\partial x}\right)_{x=x_{1}}
$$

auf der Stoßlinic $0 \leqq y \leqq l_{1}$ darstellen ]äßt. Jiese let\%tero Aussage gilt übrigons, wio man zejgen kann, auch für instationäre Strömungsfolder.

Dic Rolation (30) stellt cino nichtlineare Integralgleichung zur Berechnung des Druckfeldes $p(x, y)$ in cler Umgebung eines Flügelprofils in gashaltjgor Wasserströmung dar. Der Zusammenhang zwisehen $p$ und der Druckfunktion 60 ist dabei durch Formel (19) gegeben. ${ }^{3}$ )

2) Jeweis der Wormeln (29) durch partielle Integration und folgende 'latsachen: Die 1)iskontinuitäten müssen an den Enden der Integrationsbereicho verschwinden; In $r$ genügt der LAILAcFschen Gleichung; lïngs der Stoßlinie ist $\partial \Phi / \partial \eta=0$.

3) Die Umkehrung der Formel (19), also die J3erechnung von $p / p_{\infty}$ bei bekanntem 6 - $V^{\dagger}$ ert nuß für allgemoine $\lambda$ - Worto numerisch orfolgen; speziell bei $\lambda=1$ ergibt sich oxplizit

$$
\frac{p}{p_{(x)}}=\frac{1}{2}\left(\xi-\alpha_{\infty}\right)+\sqrt{\alpha_{\infty}+\frac{1}{4}\left(y-\alpha_{\infty}\right)^{2}} .
$$

Für dic praktisch vorkommenden Worte $\alpha_{\infty} \approx 10^{-1}$ ist der. Unterschied zwischen $b$ und $p / p_{\infty}$ schon für $p / p_{\infty} \geq 0,05$ recht unbedeutend, so gilt:

$$
\alpha_{\infty}=10^{-4} ; \quad p / p \infty=0,050 ; \quad \delta=0,048 .
$$


Die Lösung der Integralgleichung (30) erfolgte mit einem Iterationsverfahren, beginnend mit dem in. kompressiblen ])ruckfeld $p_{0}$. Tetzteres ist lediglich durch die ersten boiden Integralterme in Gleichung (30) bestimmt und kann nach Vorga be von Djekenlinie $Y_{D}$, Skelettlinie $Y_{S}$ und Anstellwinkel $\delta_{0}$ nach den bekannten Methoden der Profiltheorie für inkompressible Strömung [7] lejeht berechnet werden. ${ }^{4}$ )

Bei entsprechend großen Anstellwinkeln $\delta_{0}$ oder Zuströmgeschwindigkeiten $u_{\infty}$ können sich dabei örtlieh auch negative Druckwerte crgeben. Fs ist dann physikalisch realistisch und wio sich gezeigt hat auch für die numerische Durchführung des Itorationsverfahrens zweckmüßjg, alle Druckworto $p$, welche klciner als der Dampfdruck $p_{v}$ sind, a uf diesen Wert $p_{v}$ heraufzukorrigieren. Unter Verwendung des so bestimmten inkompressiblen Druekfeldes $p_{0}(x, y)$ bzw. $\oint_{0}(x, y)$ läßt sich das Doppolintegral über den Bereich (23) in Formel (30) berechnen. ${ }^{5}$ ) Ls liefort einen Beitrag zum Feld, den wir mit $b_{S f}^{(0)}$ bercichnen wollen.

Auf Grund der damit vorliegenden . Druckfunktion $\gamma_{0}+\delta_{f s}^{(0)}$ und des zugehörigen Truckfelds läßt sich feststellen, ob und in welchen Bereichen Uberschallzustand auftritt. Dazu braucht ja mit IIilfo der Schallgeschwindigkejt $c$ gemäß Formcl (10) nur die örtliche MACHzahl

$$
M=M_{n}=\frac{1}{c}\left(u_{\infty}+\frac{\partial \Phi}{\partial x}\right)=\frac{u_{\infty}}{c}\left[1+\frac{p_{\infty}}{\varrho_{W} u_{\infty}^{2}}(1-\gamma)\right]
$$

jedes Feldpunktes $(x, y)$ ausgerenhet zu werden. Jie $y$-Komponente der Geschwindigkeit hat dabei effektiv keinen Einfluß.

Wenn im Strömungsfeld überall Unterschallzustand herrscht, so stellt

$$
f^{(1)}=\delta_{0}+\delta_{f f}^{(0)}
$$

bereits die ersto Näherung für die Druckfunktion der gashaltigen Wasserströmung dar.

Tritt dagegen ein lokales ${ }^{6}$ ) Gebiet mit Uborschallzustand auf, das mit einem Verdichtungsstol3 abgeschlossen wird ${ }^{7}$ ), so muß noch der Anteil des Stoßintegrals in Gleichung (30) bei der Berechnung der Druck. funktion $\wp$ berücksichtigt werden. Finc crste Näherung für die Stoßlänge orgibt sich aus der Erstreckung des Überschaligebictes von $\oint_{0}+\oint_{\delta \zeta}^{(0)}$ in $y$-Richtung, wir bezeichnen sie mit $l_{1}^{(1)}$.

Die Stoßstärke $\partial(\delta)_{x=x_{1}}$ läßt sich aus den Stoßgleichungen (I7) und (18) bestimmen, wenn dor Zustand vor dem Stoß bekannt ist. In der Regel wird man in guter. Näherung davon ausgehen können, daß $\tilde{p} \approx p_{v}$, also gleich dem J)ampfdruck ist. Der zugehörige Wert von $\tilde{\delta}$ folgt aus Gleichung (19). Mit $\hat{\alpha} / \tilde{\alpha}$ aus Gleichung (18) und wegen $\hat{\jmath}=\hat{p} / p_{\infty}$ folgt:

$$
\partial(\zeta)_{x=x_{1}}=\hat{\delta}-\tilde{\delta}=\frac{\tilde{p}}{p_{\infty}} \frac{\tilde{M}^{2}}{1+\lambda}\left(1-\frac{\hat{\alpha}}{\tilde{\alpha}}\right)+\frac{\alpha_{\infty}}{\lambda}\left[\left(\frac{p_{\infty}}{\tilde{p}}\right)^{\lambda}-1\right] .
$$

$\tilde{M}$ ist die Macizzahl des Strömungszustands vor dem Stoß und ergibt siçh aus Gleichung (34).

Es bleibt nun noch die Lage $x=x_{1}$ des Stoßes am Profil zu bestimmen. Diese Aufgabe ist bereits für die schallnahe Gasströmung mehrfach diskutiert worden (vgl. z. B. [9], [10]).

Eine physikalisch korrekte Lösung ergibt sich aus der Bedingung, daß der durch den Verdichtungsstoß am l'rofil hervorgerufene Druckwiderstand gleich demjenigen Widerstand sein mul, der der Entropiezunahme im Verdichtungsstoß entspricht. In reibungsfreier Unterschallströmung verschwindet ja bekanntlich der Druckwiderstand eines Profils. Jedoch bereitet die genügend genaue Berechnung des Druckwidcrstands in Abhängig. keit von möglichen Stoßlagen $x_{1}$ erhebliche numerische Schwierigkeiten. Dahor wird in der Gasdynamik meist eine mehr heuristische Methode zur Bestimmung des $x_{1}$-Wortes angewendet; $z u m$ Bcispiel die Festlegung, daß das Druckfeld hinter dem. Stoß wieder mit der auf Grund der PRANDrsischen Regel für Unterschallströmung ermittelten Verteilung übereinstimmen soll [10]. Auch wir verwenden hier eine ähnliche heuristische M.ethode: Und zwar wird dic Stoßlinie so gelegt, daß vor dom $S$ toß in der Nähe der Profilsaugsoite, also für $x \leqq x_{1}$ und $y \geqq 0$, überall entwedor etwa Dampfdruck $p_{v}$ oder der sich sonst auf Grund des Feldes $\wp_{0}+f_{0 j}(0)$ ergebendo Minimaldruck $p_{\mathrm{SII}}>p_{v}$ herrscht. Die numerische Auswertung hat gezeigt, daß dieses Kriterium eine recht

4) Alus $Y_{s}$ und $\delta_{0}$ folgt jn unmittelbar die Wirbeldichte $\%$, also wcgen (32) auch $\partial(\zeta)_{0}$. Für Aufpunkte am Profil, also $y=0$ muß in unmittelbarer Umgebung der Vorderkante bei $x \geqq-a$ die Tangentialgeschwindigkeit $u_{\infty}+\partial \Phi / \mathrm{C} x$ noch wie üblich [7] mit dem RivGLLsfaktor $\left[1+Y_{D}^{\prime \prime 2}\right]_{2}^{-1}$ korrigiert werden.

5) Für die Auswertung des Doppelintegrals in Formel (30) hat es sich als 7weckmäßig erwiesen, zuerst bei konstantem $\eta=\eta_{n}$ über $\xi$ zu integrieren und dann die einzelnen Teilintegrale über $n$ zu summieren. I Hür $\eta_{n}=y$ tritt bei der Integration über $\xi$ bei $\xi=x$ oine Singularität auf, die sich als CAUCH Yscher Hauptwert integrieren läßt.

Es zeigt sich im übrigen, daß nur dio Bereiche niedrigen Drucks auf der Saugseito in der näheren Umgebung dos Profils einen l3eitrag von J3edeutung zum Wert des Doppelintegrals liefern. Dadurch kann der Integrationsbereich relativ klein ge. halten werden.

6) In einigem Abstand vom Profil herrscht a uf jeden Fall Unterschallzustand.• Denn bei normalem Atmosphärendruck ist der Gasvolumgehalt dort gering, $\alpha_{\infty}<10^{-3}$ und die zugehörige Schallgeschwindigkoit $c_{\infty}>200 \mathrm{~m} / \mathrm{scc}$. Eine so hohe An. strömgeschwindigkeit $u_{\infty}$ wird von Strömungskörpern im Wasser aber bei weitem nicht erreicht.

7) Aus der Gasdynamik ist bckannt, daß ein stoßfreier Ubergang von Uberschall- zu Unterschallzustand ohne zusätz. liche Strömungsbecinflussung als stabiler Vorgang nicht möglich ist [8]. Auch in der gashaltigen Vasserströmung werden, wie Experimente zeigen, Uberschallgebiete durch Verdichtungsstöße abgeschlossen. [11]. 
genaue Aussage über die Stoßlage $x=x_{1}$ liefert. $\left.{ }^{8}\right)$ Janit ist auch der Anteil des Stoles zum Feld frermittelt; wir wollen diesen mit der $\Delta$ bkürzung fst bezeichnen.

Der Ausdruck

$$
\gamma^{(1)}=\gamma_{0}+\gamma_{f f}(0)+\gamma_{\mathrm{st}}^{(0)}
$$

stellt somit die erste Xäherung für dio gesuchte Druckfunktion beim Auftreten eines lokalen Uberschall. gebietes dar.

Wenn wir zunächst die IYrnge zurïckstellen, inwieweit dic Strömungsrandbedingmer am Profil auch in der gashaltigen Strömung noch erfüllt ist, (für die inkompressible Strömung war dieses ja durch die entsprecherude Festlegung von $\partial(b)_{y=0}$ der liall; vgl. auch /iff. 4), so kömnen in ganz analoger lWeise weitere Nälerungen in der Fon

$$
\zeta^{(n)}=\wp_{0}+\jmath_{f f}^{(n-1)}+\wp_{s t}^{(n-1)} \quad(n=2,3, \ldots)
$$

berechnet werden. Dabei ist jeweils im Doppolintegral das Druckfeld der vorhergehenden Näherung einzusetzen. Außerdem sind nach den gleichen Kriterien wie bei der Ausgangsnäherung die Stoßlänge $l_{1}$. Stolßage $x_{1}$ und Stoß-Stärke $\partial(\delta)_{x=x_{1}}$ jedesmal neu $z u$ überprüfen. J)as Tterationsverfahren wird abgebrochen, wemn zwei ntufeinander folgcnde Näherungen sich effektiv nicht mehr unterscheiden.

\section{1)jo Strömungstandbedingung am Flïgelprofil}

Boi d(r. B3eschreibung des Iterationsverfahrens zur Berechnung des Druckfeldes haben wir in Ziff. 3 eine Untersuchung der Strömungsrandbedingung am l'rofil bewußt zurücküestellt, um den Gedankengang nicht zu selı' z. komplizieren. Die in cliesen Zusammenhang notwendigen Überlegungen sollen nun nachgeholt werden. Unter Berïcksichtigung des Gasgehaltes im Wasser, also mit $\left.\frac{1}{u_{(x)}} \frac{\partial \Phi}{\partial y}\right|_{y=0}$ gemäß Jormel (31) und Beachtung
der Relation (32), lautet dis: Randliedingung:

$$
-u_{\infty} Y_{*}^{\prime}(x)+u_{n}\left(\delta_{0}+\delta_{a}\right)=\frac{1}{2 \pi} \int_{-a}^{n} \frac{\gamma(\xi)}{x-\xi} d \xi
$$

In Gl. (36) ist $Y_{\delta}(x)$ die Profilskelettlinie und $\delta_{\alpha}$ cine $A$ bkürzung für dic durch dic Kompressibilität gashaltigen Wasscrs cinschließlich Verdichtungsstoß gegenïber der klnssischen Form für inkompressible Strömung zusätzlich auftretenden 'l'erme, nämlich

$$
\delta_{s}=\frac{1}{9 \pi} \frac{p_{\infty}}{\varrho_{W} u_{\infty}^{2}} \int_{0}^{l_{1}} \frac{\partial(\xi)_{\xi}-x_{1} \eta d \eta}{\left(u-x_{1}\right)^{2}+\eta^{2}}-\frac{1}{2 \pi} \int_{(B)} \int_{0} \frac{\partial}{\partial \xi} \ln \left[1+\alpha_{\infty}\left(\frac{p_{\infty}}{p}\right)^{2+1}\right]\left(\begin{array}{c}
\eta d \eta d \xi \\
(n-\xi)^{2}+\eta^{2}
\end{array}\right.
$$

In der Regel wird man davon auszugehen haben, daß die Skclettlinie des l'rofils fest vorgegeben ist. Machon wir dann für die W'irbeldichte y den Ansat'\%

$$
\gamma(x)=\gamma_{0}(x)+\gamma_{a}(x)
$$

mit $\gamma_{0}$ als der Verteilung für inkompressible St rönung, so erhalten wir für dic Berechnung der Zusnt z\%irkulation $\gamma_{a}$ die Integralgleichung

$$
u_{a} \lambda_{a}(x)=\sum_{2 \cdot \tau}^{1} \int_{-a}^{n} \frac{\gamma_{a}}{x-\frac{\xi}{\xi}} d \xi
$$

$\delta_{\alpha}$ kam als eine Art wusitzlicher, durch dic Kompressibilität bodingter Anstollwinkel interpretiert werelen, der allerdings über die Profiltiefe stark veränderlich ist.

Bei numerischen Untersuchungen und Abschäztungen hat sich gezeigt, daß der erste durch den Stoß bedingte Term in (37) den wesentlichen Anteil von $\delta_{x}$ licfert, während das Dopjelintegral nur einen unbedeutenden Einfluß hat. Im Rahmen dieser Betrachtung können wir unbedenklich $\partial(\zeta)_{5}-x_{3}$ als konstant annehmen und crhalten so

$$
\delta_{a} \approx \frac{1}{4 \pi} \frac{p_{\infty}}{Q_{s} u_{\infty}^{2}} \partial(j)_{x_{1}} \ln \left(1+\frac{l_{1}^{2}}{\left(x-\frac{\left.x_{1}\right)^{2}}{2}\right)} .\right.
$$

$-\quad-$

9) Der Wort $\wp_{3 t}$ des Stoßintegrals in Gl. (30) füllt bei festem $y\left(0<y<l_{1}\right)$ mit zunehmendem $x$ für $x<x_{1}-0$ laufend $\mathrm{ab}$ und erreiclit bei $x=x_{1}-0$ sein Minimum. $A m$ Stoß nimmt $\oint_{\mathrm{st}}$ sprunghaft um den Wert $\partial(\zeta)_{x_{1}}$ zu und fällt hinter dem Stoß für $x>x_{1}+0$ wieder kontinuierlich ab. Auf Grund dieser ligenschaften ist es möglich, $x_{1}$ so festzulegen, da $B$ in guter $\times a ̈ h e$. rung in einem gewissen Jkereich $x \leqq x_{1}$ vor dem Stoß $\gamma_{0}+\delta_{f f}^{(0)}+f_{S t}^{(0)} \approx \delta_{v} b a w . \approx b_{s u n}$ bleibt. (Vgl. auch die lirgebnisse der Beispiele in Kiff. 5.) 
Fs zeigt $\operatorname{sich}^{9}$ ), daß die Funktion $\delta_{\alpha}$ nur in einem im Verhältnis zur Profilticfe kleinen Bereich um die Stelle $x=x_{1}$ herum überhaupt merkliche Werte annimmt. Für $x=x_{1}$ wird $\delta_{a}$ logarithmisch singulär ${ }^{10}$ ), bleibt aber quadratisch integrabel. Infolgedessen existiert die Lösung der Integralgleichung (3S). Ihre effelitive und genaue Bercchnung stößt aber wegen der sehr langsamen Konvergenz ciner Fourierschen Reihenentwicklung für die Funktion $\delta_{\alpha}$ auf große Schwierigkeiten.

Man kann sich jedoch relativ leicht einen Uberblick über den qualitativen Verlauf der Lösung $\gamma_{\alpha}$ von (38) verschaffen. Diese muß so geartet sein, da $\beta \gamma_{\alpha}$ praktisch verschwindet, wenn $x$ genügend weit von der singulären Stelle $x_{1}$ entfernt ist; in der Ungebung der singulären Stelle $x=x_{1}$ mul $\gamma_{\alpha}$ so beschaffen sein, daß für $\delta_{a}$ die logarithmische Singularität herauskommt. Außerdem ist $\delta_{x}$ überall positiv. IVie ınan sich leicht klarmacht, würde dieses genau einer Diskontinuität von $\gamma_{a}$ bei $x=x_{1}$ entsprechen, es müßte daher gelten:

$$
\gamma_{\alpha}(x)=\left\{\begin{array}{l}
\text { positiv für } x=x_{1}-0, \text { und schmell auf Null abnehmend für } x<x_{1}, \\
\text { negativ für } x=x_{1}+0, \text { und schnell auf Null zunehmend für } x>x_{1} .
\end{array}\right.
$$

Für die tatsächliche numerische Berechnung der Zusatzwirbclbelegung werden wir eine andere nachfolgend erläuterte mehr physikalische Überlegung heranzichen. Dabei zeigt es sich, dab der auf diese Wcise ermittelte Funktionsverlauf von $\gamma_{a}(x)$ qualitativ weitgehend mit der oben gewonnenen Aussage übereinstimmt. Lediglich tritt an die Stelle der mathematischen Diskontinuität bei $x=x_{1}$ ein steiler Abfall der Funktion $\gamma_{a}$ in der Umgebung dieser Stelle (vgl. z. B. Bild 10).

Bei der eben erwähnten durch die physikalischen Eigenschaften des Druckfeldes gestützten Uberlegung handelt es sich um folgendes: Im Rahmen der Berchnung des Druckfeldes auf der Profilsaugseite stellt sich (unter Vorwegnahme späterer Ergebnisse) heraus, daß der Winfluß der Kompressibilität sich im wesentlichen auf das Uberschallgebiet vor dem Verdichtungsstoß und den Bereich unmittelbar hinter dem Stoß erstreckt. In der direkten Ungebung des Staupunktes an der Vorderkante sowie in dem Gebiet in einigem Abstand (etwa $10 \%$ der Flügeltiefe) hinter dem Stoß ist der Strōmungszustand absolut inkompressibel. Fs erscheint somit logisch, daß das Strömungsfeld auch auf der gesamten Druckseite inkompressiblen Charakter haben muß. Denn bei der hier behandelten stationären ebenen Profilströmung ohne Berücksichtigung des Einflusses der Flügelseitenkanten ist nicht. einzusehen, daß sich die Kompressibilität a uf der Druckseite in Anbetracht der dortigen kleinen, einer rein inkompressiblen Strömung entsprechenden örtlichen Mach\%ahlen bemerkbar machen kann.

Die Bedingung, daß clas Feld fo auf der Druckseite des Profils den inkompressiblen Zustand beibehalten muß, läßt sich nun in einfacher Weise zur Bestimmung der Zusatzwirbelbelegung $\gamma_{\alpha}$ verwenden. Dazu berechnet man mit dem in Ziff. 3 dargestellten Iterationsverfahren den Verlauf der Druckfunktion $\wp(x,+0)$ auf der Saugseite zunächst unter Beibehaltung des inkompressiblen Ausgangswertes von ${ }^{11}$ )

$$
\partial(\zeta)_{y=0}=-\frac{\varrho_{w} u_{\infty}}{p_{\infty}} \gamma_{0}(x)\left[1+\gamma_{i}^{\prime 2}\right]^{-1} \text {. }
$$

Jabei zeigt es sich, daß durch den Winfluß ales StoBintegrals und Doppelintegrals in (30) das Feld 60 auf der Druckseite, also

$$
\wp_{*}(x,-0)=\hat{\wp}(x,+0)+\frac{\Omega_{W} u_{\infty}}{p_{\infty}} \gamma_{0}(x)\left[1+Y_{D}^{\prime}\right]^{-1}
$$

in der näheren Umgebung der Stoßlage $x=x_{1}$ doch merklich von der inkompressiblen Verteilung $\oint_{0}(x,-0)$ abweicht. Dic Berlingung

$$
\oint_{0}(x,-0)=\mathfrak{\wp}(x,-0)
$$

läßt sich jedoch durch Finführung der Zusatzbelegung $\gamma_{a}$ erfüllen. Es muß dann

$$
\wp_{0}(x,-0)=\wp(x,+0)+\frac{\varrho_{w} u_{\infty}}{p_{\infty}} \frac{\gamma_{0}(x)+\gamma_{\alpha}(x)}{1+Y_{\bar{D}}^{\prime}}
$$

scin, und aus (43) und (41) folgt die gesuchte Bestimmungsgleichung für $\gamma_{a}$, welche wir an Stelle der Integralgleichung (38) verwenden:12)

$$
\frac{1}{u_{\infty}} \gamma_{a}(x)=\frac{p_{\infty}}{\varrho_{w}}\left(1+Y_{\infty}^{\prime 2}\right)\left[\wp_{0}^{2}(x,-0)-\wp_{*}(x,-0)\right]
$$

9) Diese 'Tatsache ergibt sirh unmit telbar aus Formel (39), wenn man berücksichtigt, daß in den meisten bej Anwendun. gen auftretenden fiillen gilt:

$$
l_{1}^{\prime} / a^{2} \leqq 10^{-3} ; \quad \frac{1}{4 \pi} \frac{e_{\infty}}{0 w w_{\infty}^{2}} \partial(\xi) \leqq 0,0 ; .
$$

10) Der FuBpunkt $x=x_{1}$ und $y=0$ der StoBlinie muB streng genommen als eine Art singulärer Punkt aus unseren Be. trachtungen ausgenommen werden. Denn dieser Punkt gehört gemeinsam den Randkurven $\left(c_{3}\right)$ und $\left(c_{4}\right)(v g l$. Bild 2$)$ der bei der Gewinnung von (31) und (30) verwendeten GREexschen Formel an.

11) Für $y= \pm 0$ ist wie in der Profilt heorie üblich der RifGessfaktor zu berücksichtigen.

12) Zur Auswertung der Gleichungen (41) und (44) bemerken wir noch: Das Doppelintegral in Formel (;0) ist stetig für $y=+0$; auch das StoBintegral aus (30) bleibt beim Durchgang durch die $x$-Achse stetig mit Ausnahme des $\mathrm{l}^{\text {'unktes }} x=x_{1}$. Unter $f^{*}\left(x_{1},-0\right)$ wollen wir dann das arithmetische Hittel der sich bei $x_{1}+0$ und $x_{1}-0$ ergebenden W'erte verstehen. 
Abschließend bleibt noch zu prüfen, ob und inwieweit der Einfluß von $\gamma_{x}$ das vorher nur mit $\gamma_{0}$ berechnete Feld $\delta$ auf der Saugsejte des Profils verändert. Wie sich gezeigt hat, ist die Abweichung in vielen Fällen un. erheblich, gegebenenfalls muß sie mit einer weiteren Iteration gemä 3 Ziff. 3 berücksichtigt werden.

\section{Anwendung und Ergelonisse der Theoric}

Bevor die vorgelegte Theorie auf konkrete Beispiele angewendet werden kann, müssen wir uns noch über den Exponenten $\lambda$ klar werden, der gemäß Gleichung (I) die Abhāngigkeit des örtlichen Massenverhältnis $\mu$ vom Druckfeld bestimmt. Gleichzeitig ist es auch erforderlich, einen realistischen Wertebereich für das Gasvolumenverhältnis $\alpha_{\infty}$ der Zuströmung festzulegen.

Wie wir in der Einleitung ausgeführt haben, stellt die Relation (1) nur eine heuristisch angesetzte sum. marische Näherungsformel dar und wurde nicht auf Grund von Betracht ungen einzelner physikalischer Vorgängo gewonnen. Daher wird man eine genauere Aussage über den Wert von $\lambda$ erst durch Vergleiche zwischen den Ergebnissen der 'Theorie und denjenigen eingehender darauf abgestimmter experimenteller Untersuchungen gewinnen können.

Da letztere zur Zeit nur in sehr begrenztem Umfang zur Verfügung stehen [11], müssen wir uns für die numerische Auswertung der Theorie mit einer angenäherten Festlegung von $\lambda$ begnügen. ${ }^{13}$ ) Schon auf Grund von rein qualitativen Oberlegungen erscheint es unrealistisch, $\mu$ als konstant $(\lambda=0)$ anzusetzen, wenn man mit $\mu$ sowohl den Anteil der freien Luftmasse als auch denjenigen der bei Bildung von Dampfkavitation entstehenden Thampfmasse beschreiben will (vgl. Finleitung).

Soweit es sich nur um das Massenverhältnis des freien Luftgehalts ohne Dampfkavitation handelt, wird in der Literatur überwiegend von einem konstanten $\mu$-Wert ausgegangen und damit die Schallgeschwindigkeit und andere thermodynamische Größen berechnet (vgl. z. B. [1], [2], [3], [12], [13], [14]).

Sinнax findet dagegen eine erhebliche Druckabhāngigkeit von $\mu$ mit einem Verlauf ähnlich dem Potenzgesetz (1), [15]. Auch andere funktionale Zusammenhänge erscheinen denkbar. ${ }^{14}$ )

Nun jiegt der Massenanteil an gelöster Luft im Wasser bei normalen Anströmungsdrücken von etwa $1 \mathrm{kp} / \mathrm{cm}^{2}$ in der Größenordnung von $10^{-4}$ bis $10^{-5}$. Der uns interessicrende freic Luftgehalt beträgt bei solchen Drücken sicher nur einen kleinen Bruchteil davon; nach den aus der erwähnten Literatur zu entmehmenden Angaben dürfte er ungeführ um zwei Größenordnungen tiefer liegen. Man kann somit etwa

$$
10^{-7} \leqq \mu_{\infty} \leqq \mathrm{I} 0^{-6}
$$

annehmen. Der realistische Bereich für das Volumverhältnis $a_{\infty}$ der Anströmung (die ja wegen ihres relativ hohen Druckes in der Regel noch keine Dampfkavitation enthält) wird demnach ungefähr

sein.

$$
\frac{1}{2} 10^{-1} \leqq \alpha_{\infty} \leqq 10^{-3}
$$

Bezieht man die 1)ampfkavitation in die Čberlegungen ein, so würden sich beim Sirreichen von Dampfdruck im Strömungsfelal also für $p \approx p_{v}=0,03 \mathrm{kp} / \mathrm{cm}^{2}, p_{\infty} / p \approx 33$ folgende Verte für den Iuftvolumen. gehalt $\alpha$ ergeben:

$$
\begin{aligned}
& \lambda=0: \quad a \approx \quad 33 a_{\infty} \text { oder } \quad 3,6 \cdot 10^{-3}<a<3,3 \cdot 10^{-2} ; \\
& \lambda=1: \quad a \approx 1100 a_{\infty} \text { eder } 0,06<a<1,1 \\
& \lambda=2: \quad a \approx 36000 a_{\infty} \text { oder } \quad 3,8<a<36 .
\end{aligned}
$$

Gehen wir im lahmen dieser heuristischen Betrachtung davon aus, daß für $p \approx p_{v}$ ein Wasser-Gas-Gemisch vorliegt mit einem Gasvolumenanteil a zwischen $10 \%$ und $50 \%$, so ist der Wert $\lambda=1$ am plausibelsten. Die nachfolgend mitgeteilten Zahlenergebnisse konkreter Profilströmungen wurden daher mit $\lambda=1$ berechnet. Außerdem liegt der Luftvolumengehalt $\alpha_{\infty}$ der \%uströmung in dem durch (45) gegebenen Bereich.

liei den Jeispielen 1 bis 6 handelt es sich um ein ungewölbtes $\left(Y_{*}=0\right)$ filügelprofil mit der Diokenlinie

$$
\frac{1}{a} Y_{D}=0.00 \jmath^{\prime} \overline{1-(x / a)^{2}} \text {. }
$$

Der Druck der Zuströmung ist mit $p_{\infty}=1 \mathrm{kp} / \mathrm{cm}^{2}$, der Kavitationsdruck und damit zugleich der überhaupt auftretende Minimaldruck mit $p_{v}=0.03 \mathrm{kp} / \mathrm{cm}^{2}$ angenommen. Die einzelnen Beispiele unterscheiden sich durch ihre Anstellwinkel $\delta_{0}$, das Volumenverhältnis $\alpha_{\infty}$ und den Gesehwindigkeitsbeiwert der \%uströmumg $\varrho_{w} u_{\infty}^{\prime 2} / \varrho_{\infty}$. Let\%terer stellt praktisch die reziproke Kavitationszahl dar. Die Werte sind:

$\begin{array}{llll}\text { Beispicl 1: } & \delta_{0}=0,020 ; & \alpha_{\infty}=10^{-4} ; & \varrho_{W} u_{\infty}^{2} / p_{\infty}=4,0 . \\ \text { Beispiel 2: } & \delta_{0}=0,025 ; & a_{\infty}=10^{-4} ; & \varrho_{W} u_{\infty}^{2} / p_{\infty}=4,0 . \\ \text { Beispiel 3: } & \delta_{0}=0,030 ; & a_{\infty}=10^{-4} ; & \varrho_{W} u_{\infty}^{2} / p_{\infty}=4,0 . \\ \text { Beispiel 4: } & \delta_{0}=0,025 ; & \alpha_{\infty}=1.10^{-4} ; & \varrho_{W} u_{\infty}^{2} / p_{\infty}=4,0 . \\ \text { Beispiel 5: } & \delta_{0}=0,025 ; & a_{\infty}=2 \cdot 10^{-4} ; & \varrho_{W} u_{\infty}^{2} / p_{\infty}=4,0 . \\ \text { Beispiel 6: } & \delta_{0}=0,030 ; & \alpha_{\infty}=10^{-4} ; & \varrho_{w} u_{\infty}^{2} / p_{\infty}=2,25 .\end{array}$

${ }^{13}$ ) Darunter wollen wir eine Beschrïnkung von $\lambda$ auf ganze Zahlen verstehen.

14) In einer persönlichen Mitteilung an die Verfasser gibt H. .J. BAITFR für $\mu(p)$ ohne Berücksichtigung von Dampfkavitation ein lincures Geset\% an. Fs beruht auf der Voraussetzung, daß sich lokal stets Gleichgewicht zwischen gelöster und ungelöster Luft einstellt. 


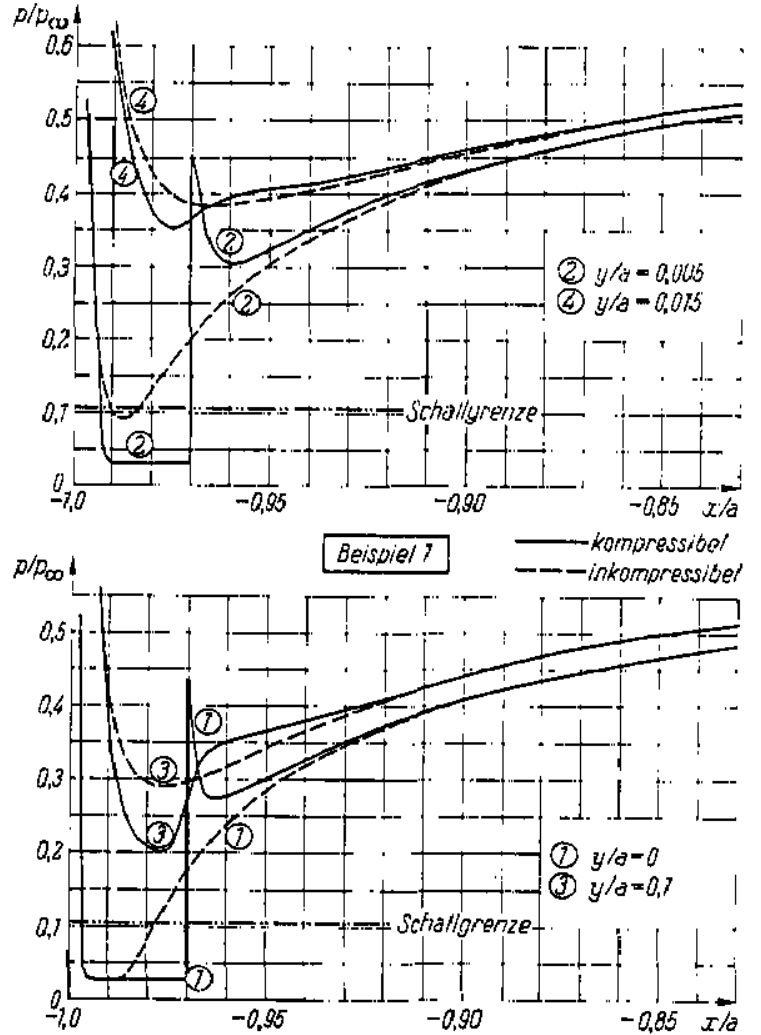

1)ild 3

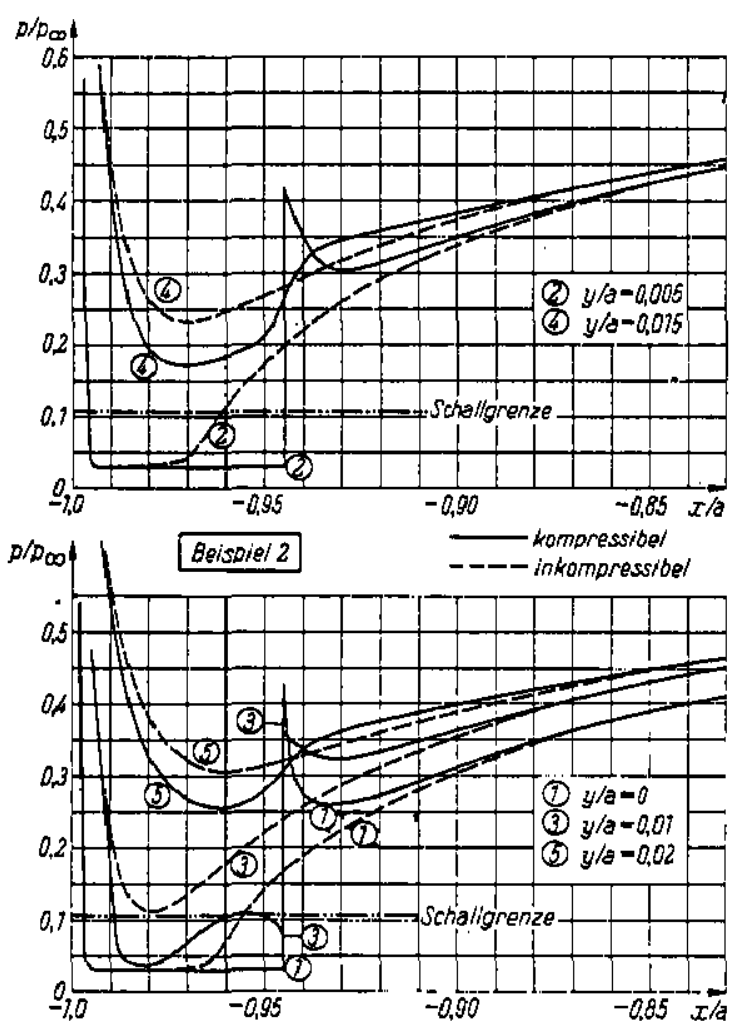

Jijla 4
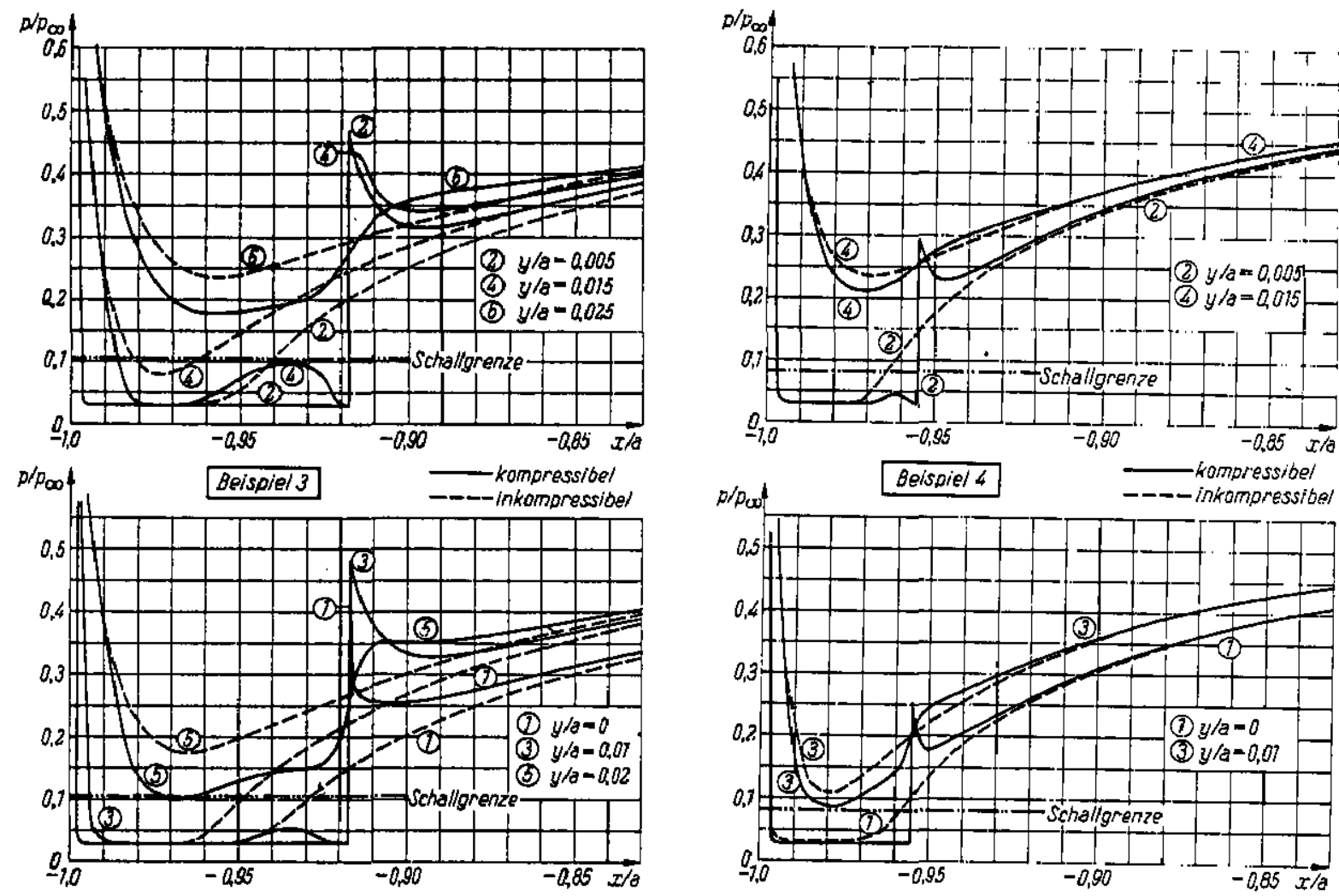

Blla 5 
])ie inkompressible Strö̈nung um das Profil ist durch the Wirbeldichte

$$
\gamma_{0}=2 \delta_{0} u_{\infty} \sqrt{\frac{a-x}{a+x}}
$$

und die Quollen-Senkenbelegung

$$
q=0,06 u_{\infty}\left[\sqrt{\frac{a-x}{a+x}}-\sqrt{\frac{a+x}{a-x}}\right]
$$

gekennzeiclnet.

Mit Jilfe des in Ziff. 3 bescliriebenen Iterationswerfahrens wurde das Druckfeld auf der l'rofilsanureito crenittelt. In allen Fällen waren drei Iterationsschritte ausrcichend, um Konvergenz a erreichen. Dis Lirgeb)nisso sind in den IBildern 3 bis $\mathbf{8}$ dargestellt. Zum Vergleich ist die Druckverteilung bei inkompressibler Strö. mung gestrichelt eingezeichnet.
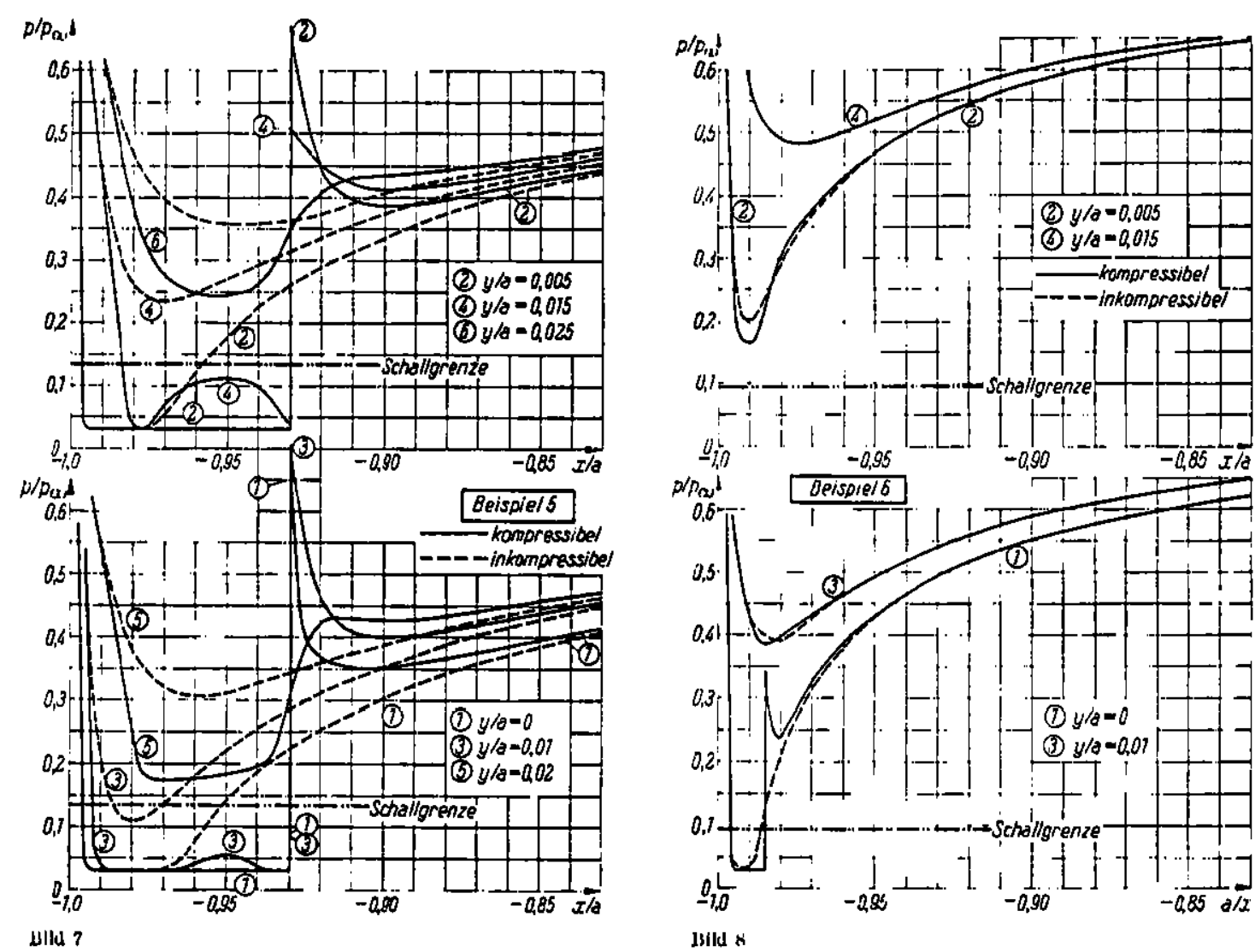

Die erhaltenen Druckverläufe zeigen doutlich, daßs der Einfluf3 dor clurch den (dangehalt bedingten Kom. pressibilität des Wassers sich lediglich auf die Ungebung des mit ejnem Verdichtungsstoß alogeschlossenen Oberschallgelietes beschränkt. Bereits kur\% hinter dem Stoß erreicht das 1)ruckfeld wiecler seinc'n inkompressiblen Zustand. Entsprechend ist es in $y$-Kichtung senkrecht zur Profilstruktur. Die Stoßlänge $l_{1} / a$ bei den einzelnen Beispielen geht aus der nachfolgenden Tabelle hervor:

\begin{tabular}{lllllllllll}
\hline leispiel & 1 & 2 & 3 & 4 & 5 & & 6 & \\
\hline$l_{1} / a$ & 0,0075 & 0,0125 & 0,0175 & 0,0075 & 0,0175 & 0,0025 \\
\hline
\end{tabular}

Nennenswer te Abweichungen vom inkompressiblen Stı ömungsfeld treten überhaupt nur auf, wemn Oberschnll. zustand erreicht wird. Selbst Beispiel 6 mit dem nur noch schwach ausgeprägten Uberschallgebjet hat schon fast inkompressible Druckverteilung. Die Verhältnisse unterscheiden sich also wesentlich von denjenigen eincrschallnahen Juftströmung sowohl mit als auch ohne lokales Uberschallgebiet. Denn bei letgterer ist ja tas gesamte Strömungsfeld auch in großem Abstand von einem eventuell auftretenden Ubersehallbereich gegenüher dem inkompressiblen Zustand erheblich modifiziert, also die MACHzahl überall entsprechend hoch.

Der Grund für dieses verschiedene Verhalten ist folgender: 13ei Luft ist die örtlicho Schallgeschwindigkeit $\sim \sqrt{T}$, bleibt also in ginzen Strömungsfeld eines schlunken Profils fast kunstant. 13ei gashaltigem Wasser 
sinkt der Wert der Schallgeschwindigkeit in den Unterdruckgebieten am Profil um weit mehr als eine Größenorłnung gegenüber dem für die in der Regel noch inkompressible Zuströmung geltenden Wert ab. Da bei einer Strōmung um schlanke Flügelprofile (mit Ausnahme der Umgebung des Staupunktes) sich (lie Geschwindigkeit im ganzen Strömungsfeld nur mäßig verändert, ist durch das Verhalten der Schallgeschwindigkeit auch die Variation der örtlichen MAcrzahl und damit der Kompressibilitätseinfluß bestimmt.

Die Ergebnisse der Beispiele 1, 2, und 3 zeigen ferner, da B bei gleicher Geschwindigkeit $u_{\infty}$ und gleichem Iuftvolumengehalt $\alpha_{\infty}$ der \%uströmung die Ausdehnung des Uberschallgcbietes und damit die Stoßlänge mit zunchmendem Anstellwinkel $\delta_{0}$ größer wird. Dagegen bleibt die Stoß-Stärke (der Drucksprung) ungefähr konstant, wenn vor dem Stoß bereits etwa Dampfdruck herrscht.

Durch Vergleich der Druckverteilungen der Beispicle 4, 2 und 5 erhält man einen Öberblick, wie sich bei sonst gleichen Parametern eine Änderung des Luftvolumengehaltes $\alpha_{\infty}$ der Zuströmung auswirkt. Sowohl die Ausdehnung des Uberschallgebietes als auch die Stoß.Stärke nehmen mit $\alpha_{\infty}$ erheblich zu; der Grenzdruck $\left(p / p_{\infty}\right)_{M=1}$, bei dem die Schallgeschwindigkeit erreicht wird, steigt ebenfalls an (vgl. auch Bild 20).

Eine Betrachtung der Resultate von Beispiel 3 und 6 vermittelt einen Eind 'uck von dem großen Einfluß, den der Beiwert $\varrho_{w} u_{\infty}^{2} / p_{\infty}$ (also et wa dic reziproke Kavitations\%ahl) auf die Forn des Druckfeldes und die Größe des Überschallgebietes ausübt.

Bild 9 zeigt der Verlauf der Druckverteilung auf der P'rofildruckseite direkt an der Kontur. Dieser stimmt voraussetzungsgemäß (vgl. Formel (42)) mit dem der inkompressiblen Strömung überein. Schließlich gibt Bild 10 die durch den Einfluß der Kompressibilität und insbesondere des Verdichtungstoßes bedingte zusätzliche Wirbelbelegung $\gamma_{\alpha}$ (bezogen a uf die Verteilung $\gamma_{0}$ der inkompressiblen Strömung) wieder. $\gamma_{\alpha}$ hat tatsächlich weitgehend den in Ziff. 4 bei der Diskussion der Strömungsrandbedingung vorhergesagten Verlauf.

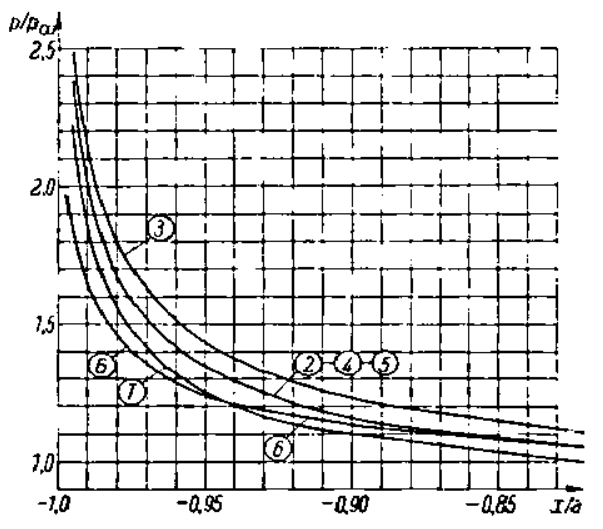

Jsild 9. Druckverteilung an der Druckseite, Beispiele 1 bis 6

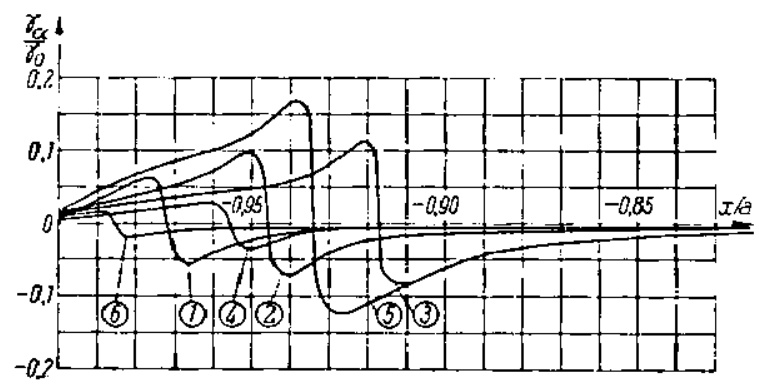

13ild 10. Zusatzzirkulation, Beispiele 1 bis 6

In allen Fällen tritt hinter dem Verlichtungsstoß zunächst ein erheblicher Druckabfall auf. Diese auch als Nachexpansion bezeichnete Erscheinung ist auch bei schallnahen Luftströmungen bekannt und experimentell bestätigt [6], [16].

Die weiteren Beispicle 7 bis 12 beziehen sich auf ein dickeres und zugleich gewölbtes Profil. Und zwar haben die Dickenlinie und die Skelettlinie die Form

$$
\frac{1}{a} \cdot Y_{D}=\sqrt{1-x^{2} / a^{2}}\left(0,10-0,07 \frac{x}{a}\right) ; \quad \frac{1}{a} Y_{s}=0,065\left(1-x^{2} / a^{2}\right)
$$

Die maximale relative Dicke liegt bei $x / a=-0,435$ und beträgt $11,7 \%$.

Der Dampfdruck und damit zugleich der überhaupt auftretende Minimaldruck ist wic vorher $p_{v}=$ $=0,03 \mathrm{kp} / \mathrm{cm}^{2}$. Der Druck der \%uströmung wurde für die Bejspiele 7 und $10 \mathrm{mit} p_{\infty}=1 \mathrm{kp} / \mathrm{cm}^{2} \mathrm{für}$ die Bei. spiele $8,9,11$ und 12 mit $p_{\infty}=0,5 \mathrm{kp} / \mathrm{em}^{2}$ angenommen. ${ }^{15}$ ) Die weiteren Daten sind:

$$
\begin{array}{llll}
\text { Beispiel 7: } & \delta_{0}=0,05 ; & \alpha_{\infty}=10^{-4} ; & \varrho_{w} u_{\infty}^{2} / p_{\infty}=2,0 \\
\text { Beispiel 8: } & \delta_{0}=0,05 ; & \alpha_{\infty}=10^{-4} ; & \varrho_{w} u_{\infty}^{2} / p_{\infty}=2,0 \\
\text { Beispiel } 9: & \delta_{0}=0,05 ; & \alpha_{\infty}=4 \cdot 10^{-4} ; & \varrho_{w} u_{\infty}^{2} / p_{\infty}=2.0 \\
\text { Beispiel 10: } & \delta_{0}=0,00 ; & \alpha_{\infty}=10^{-4} ; & \varrho_{W} u_{\infty}^{2} / p_{\infty}=3,4 \\
\text { Beispiel 11: } & \delta_{0}=0,00 ; & \alpha_{\infty}=10^{-4} ; & \varrho_{w} u_{\infty}^{2} / p_{\infty}=3,4 \\
\text { Beispiel 12: } & \delta_{0}=0,00 ; & \alpha_{\infty}=4 \cdot 10^{-4} ; & \varrho_{w} u_{\infty}^{2} / p_{\infty}=3,4
\end{array}
$$

15) Ähnliche Verhältnisse liegen bei den von Swuts [11] durchgeführten Versuchen mit einem entsprechenden Flüge] profil vor. Dort wird das Wasser von einem Ruhedruck $p_{0}$ mit $u_{0}=0$ auf die Zuströmgeschwindigkeit $u_{\infty}$ mit dem zug. Druck $p_{\infty}$ beschleunigt. Die Beispiele 8, 9 entsprechen den Daten: $p_{0}=1 \mathrm{kp} / \mathrm{cm}^{2} ; p_{\infty}=0,5 \mathrm{kp} / \mathrm{cm}^{2} ; u_{\infty}=10 \mathrm{~m} / \mathrm{sec}$. Die Beispiele 11, 12 ent sprechen den Werten: $p_{0}=1,35 \mathrm{kp} / \mathrm{cm}^{2} ; p_{\infty}=0,5 \mathrm{kp} / \mathrm{cm}^{2} ; u_{\infty}=13 \mathrm{~m} / \mathrm{sec}$. Der Luftwolumengehalt im likuhekessel $\alpha_{0}$ beträgt $10^{-4}$ für die Beispiele 9 und 12 und $0,25.10^{-4}$ für die Beispiele 8 und 11 , wenn auch für die Beschleunigung aus dem Ruhekessel heraus Gleichung (1) mit $i=1$ zugrunde gelegt wird. 


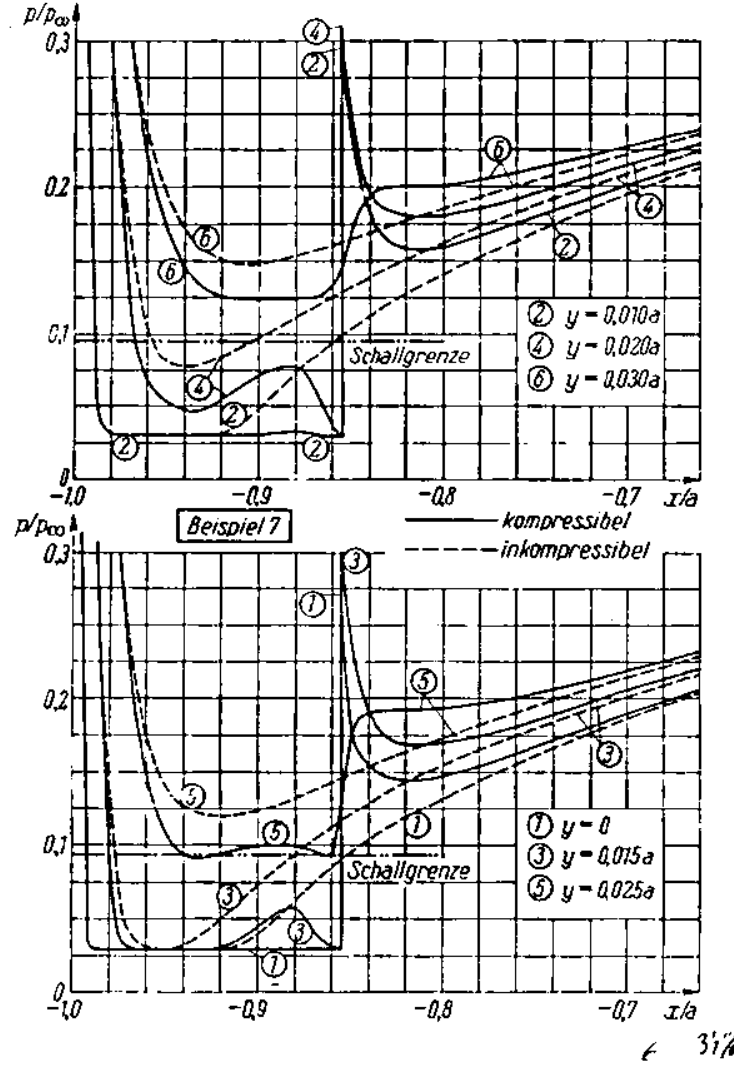

Bild 11

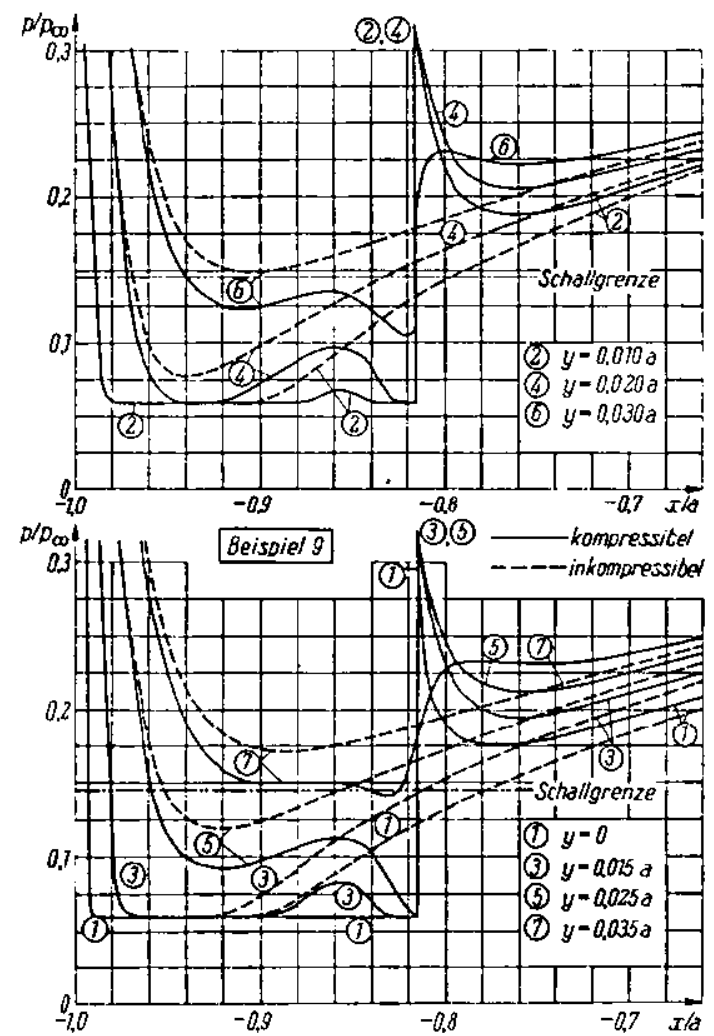

Bild 13
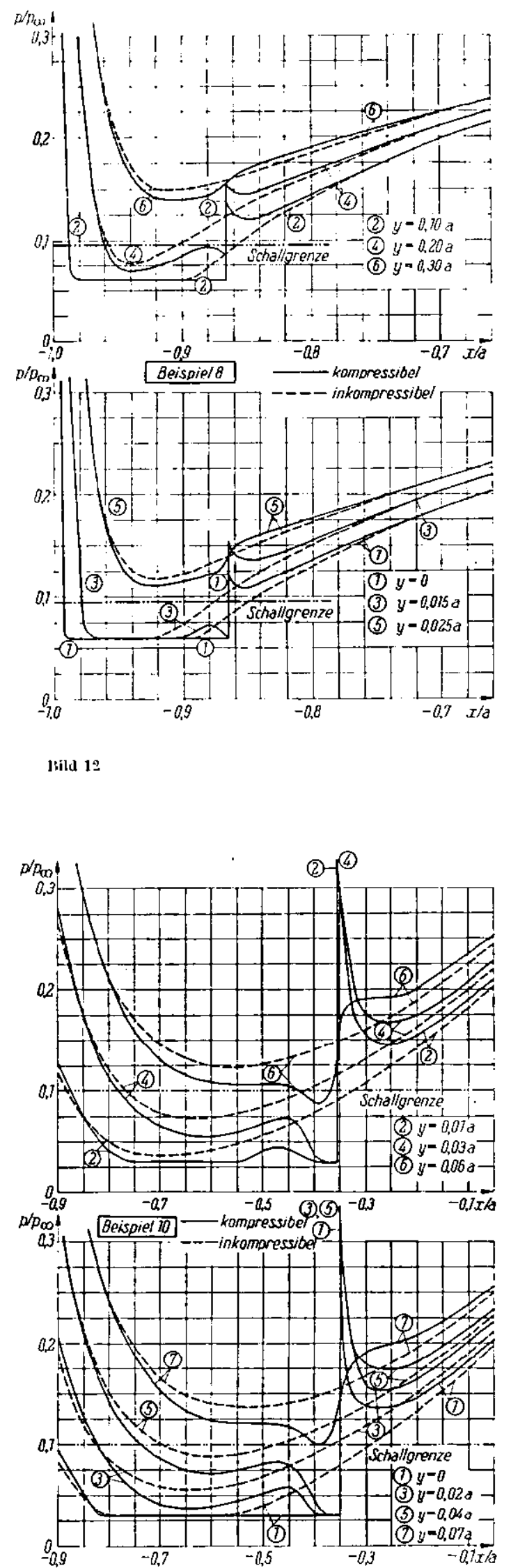

Bitl 14 
Die inkompressible Strömung um das l'rofil ist durch die Wirbeldichte

$$
\gamma_{0}=2 u_{\infty}\left[\delta_{0} \sqrt{\frac{a-x}{a+x}}+0,13 l^{\prime} \overline{1}=\left(x^{2} / \overline{\left.a^{2}\right)}\right]\right.
$$

und die Quellen-Senkenbelegung

$$
q=u_{\infty}\left[0,17 \sqrt{\frac{a-x}{a+x}}-0,03 \sqrt{\frac{a+x}{a-i c}}-0,28 \sqrt{1--\left(x^{2} / a^{2}\right)}\right]
$$

bestimnt.

Die Ergebnisse für den Verlauf der Druckverteilung auf der Profilsaugseite sind in den Bilder 11 bis 16 dargestellt. Dic inkompressiblen Druckwerte sind zum Vergleich wieder gestrichelt mit eingetragen. Dio Stoßlängon $l_{1} / a$ sind in der nachfolgenden Tabelle zusammengestellt:

\begin{tabular}{lllllll}
\hline Boispiel & 7 & 8 & 9 & 10 & 11 & 12 \\
\hline$l_{1} / a$ & 0,0225 & 0,0225 & 0,030 & 0,050 & 0,045 & 0,090 \\
\hline
\end{tabular}

T)ie bercits bei dor Diskussion der Ergobnisse von T3eispiel 1 bis 6 hervorgehobenen Wigensohaften des 1)ruckfeldes sind grundsätzlich auch bei den neuen Beispielen 7 bis 12 vorhanden; wir brauchen daher im folgenden nur noch auf einige physikalische Besonderheiten hinweisen.

So ist es verständlich, daß bei dem (gegenüber IBoispiol 1 bis (6) dickeren und außerdem gewölbten Profil für Anstellwinkel $\delta_{0}=0,05$ ausgcpräugte Oberschallıustände schon bei den relativ kikinen Beiwert $\frac{\varrho_{W} u_{\infty}^{2}}{p_{\infty}}=2$ auftreten. Das lokale Oberschallgebiet ist sowohl stromabwärts als auch senkrecht zum Drofil weiter ausgedehnt. (Beispiel 7 bis 9). Ohne Anstellwinkel, also für $\delta_{0}=0$, wäre die Strömung für $\frac{\varrho_{w} u_{\infty}^{2}}{p_{\infty}}=2$ noch rein inkompressibel; orst oberhalb von $\frac{\varrho_{w} u_{\infty}^{2}}{p_{\infty}}=3$ (für Beispiel 10 bis 12 wurdo der Wert 3,4 gowählt) treten lokale Utberschallgebiete auf, deren Ausdehnung dann allerdings wesentlich größer ist als bei dem entsprechen. den Fall mit Anstellwinkel. J.etztere Tatsache ist durch das stärkerc radialo Druckgefälle der durch den Ein. fluß des Anstellwinkels gekrümmten Stromlinien bedingt.

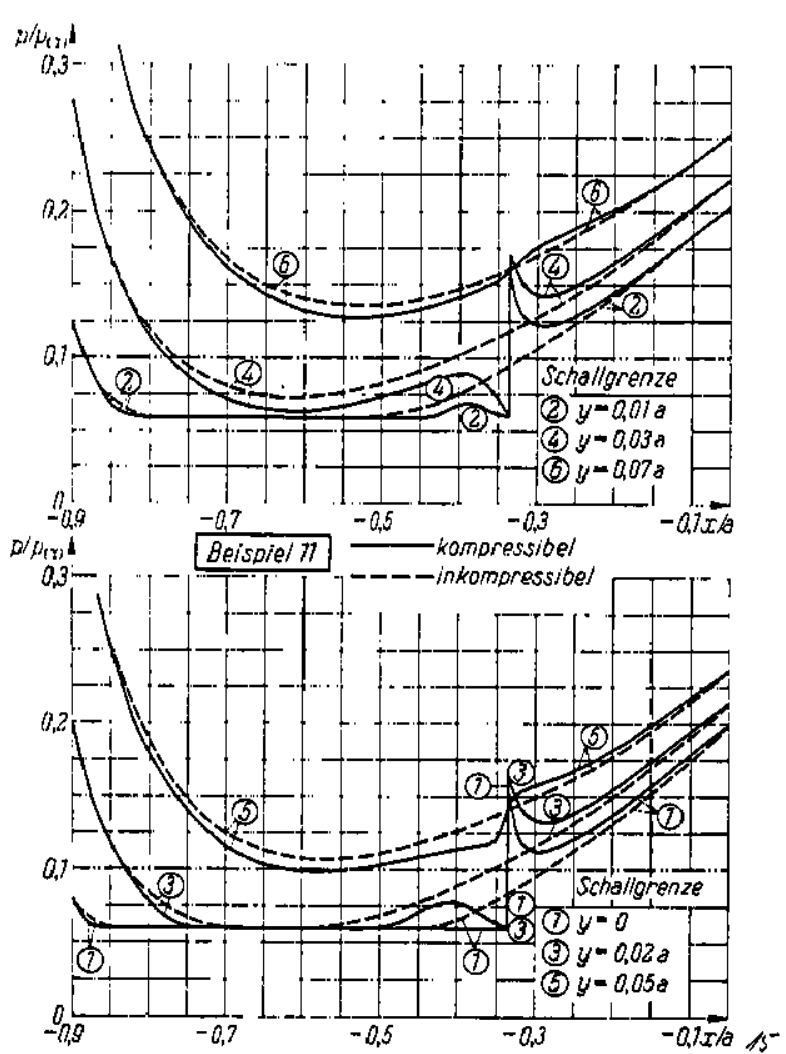

Bild 15

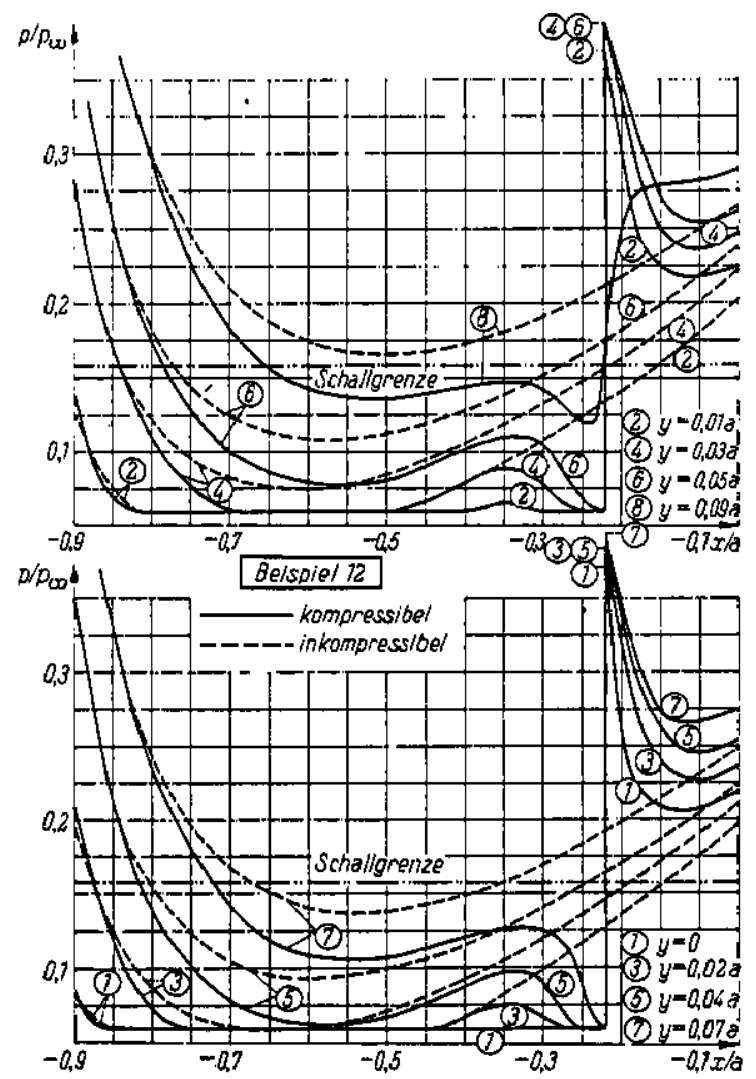

bild 10 
Ein Vergleich von Beispiel 8 mit 9 sowie von 11 mit 12 zcigt wieder starken Einfluß des Gasvolumengehalts $\alpha_{\infty}$ der Zuströmung.

Eine Betrachtung der Beispiele 7 und 9 sowie 10 und 12 mit dem jeweils gleichen Beiwert $\varrho_{W} u_{\infty}^{2} / p_{\infty}$ und übcreinstimmendem $\alpha$-Wort für $p=1 \mathrm{kp} / \mathrm{cm}^{2}$ gemäß Formel (7) zeigt: Wenn bereits bei $p=1 \mathrm{kp} / \mathrm{cm}^{2}$ dis Zuströmgeschwindigkeit zum P'rofil vorhanden ist, (Boispiel 7 und 10), so wird die Stoß.Stärke größor aber die Ausdehnung des Überschallgebietes kleiner als wenn bei $p=1 \mathrm{kp} / \mathrm{cm}^{2}$ noch Ruhezustand herrscht und die Zuströmgeschwindigkeit $u_{\infty}$ erst bei dem niedrigeren Druck $p=0,5 \mathrm{kp} / \mathrm{cm}^{2}$ erreicht ist; (Beispicl 9 und 12).

Geht man anderersoits von gleichen $\alpha_{\infty}$.Werten und gleichen Kavitationszahlen (d. h. etwa auch gleichem $\left.\varrho_{w} u_{\infty}^{2} / p_{\infty}\right)$ für den Zuströmzustand $p_{\infty}, u_{\infty}$ aus, so bleibt die Ausdehnung eines etwa auftretenden Uber'schall. gebietes annähernd konstant, während die Stoßstärke mit zunehmenclem $p_{\infty}$, bzw. $u_{\infty}$ erheblich zunimmt. Dioses geht aus einem Vergleich von Beispicl 7 mit 8 sowie von $10 \mathrm{mit} 11$ hervor. Dic Lnergie der Zuströmung ist ja auch bei $8 \mathrm{bzw} .11$ wesentlich niedriger als boi 7 bzw. 10 .

IIinter dem Verdichtungsstoß tritt auch bei Beispiel 7 bis 12 dic bekannte Nachexpansion (also Druck. abfall) auf.

In Bild 17 ist dio Druckverteilung am Profil auf desson 1)ruckscite dargestellt. Wie in Ziffer 4 dargelegt wurde, ist sie mit dorjenigen der inkomprossiblen Strömung identisch. Dio IBilder 18 und 19 zeigen die dureh den Einfluß der Kompressibilität einschließlich des Verdichtungsstoßes bedingte zusätzliche Zirkulationsver. teilung $\gamma_{\alpha}$ und zwar bezogen auf die Belegung $\gamma_{0}$ des l'rofils bei inkompressibler Strömung. Der Verlauf von $\gamma_{a}$ entspricht wieder weitgehend den Ǔberlegungen aus Ziffer 4.

Unabhängig von den oben diskutierten Ergebnissen der behandelten speziellen Beispiele 1 bis $12 \mathrm{kommen}$ wir nun noch zu einigen allgernoineren Betrachtungen.

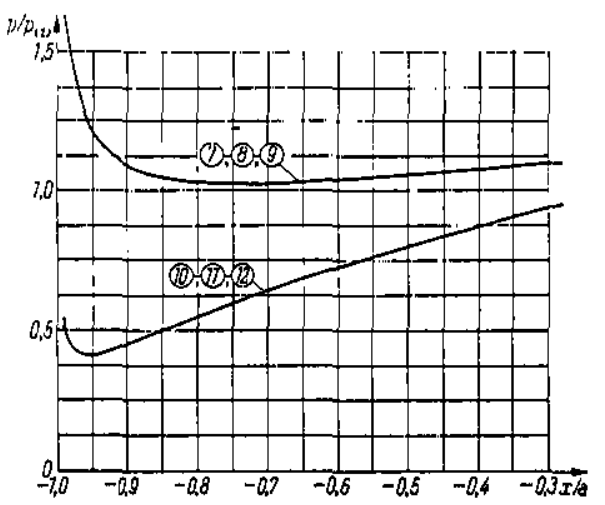

13ild 17. Druckvertcilung an der Druckseite, Jeisplelo 7 bis 12

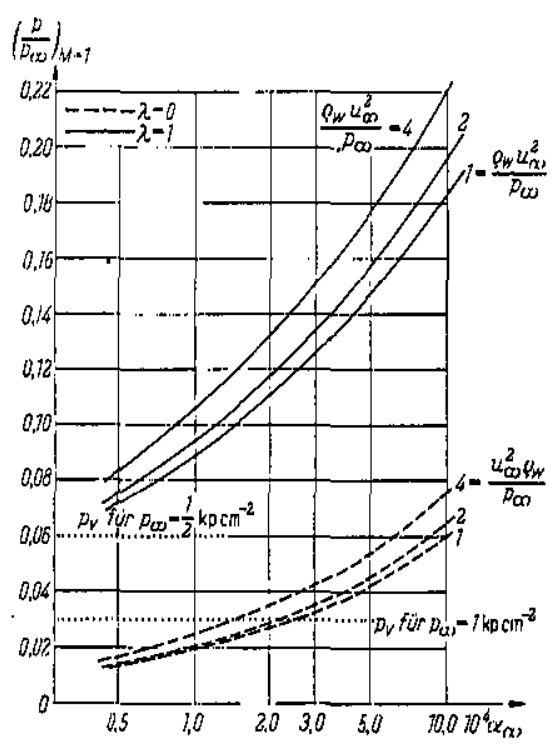

bild 20

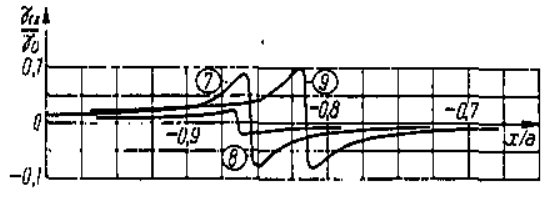

13ild 18. Zusatzzlrkulation, Bolsplele 7-9

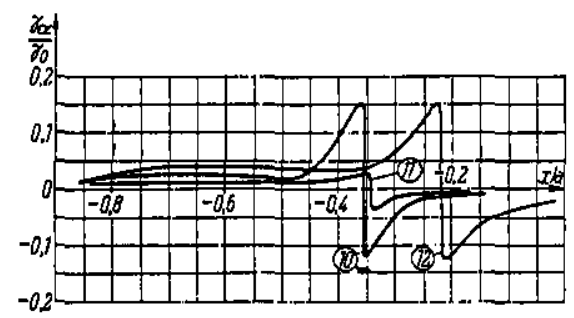

Bild 19. Zuaratzickulation, I3elspicle 10-12

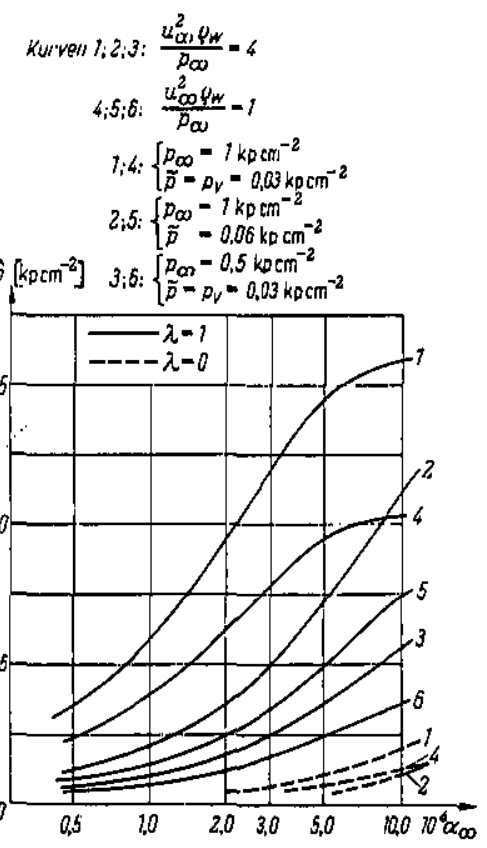

Blld 21 
In Bild 20 ist in $\Lambda$ bhängigkeit von den Zuströmungsparametern $\alpha_{\infty}$ und $\varrho_{W} u_{\infty}^{2} / p_{\infty}$ der Druck $\left(p / p_{\infty}\right)_{M=1}$ dargestellt, bei dem im Strömungsfeld Überschallzustand erreicht wird. Dicser J)ruck wächst verständlicher. weise mit den beiden genannten Parametern. Zum Vergleich sind gestrichelt dicjenigen Werto eingezeichnet, welche sich für cin konstantes Massenverhältnis $\left(\lambda_{*}=0\right)$ ergeben würden. Man erkennt, da.B sich im Gegensatz zu experimentellen Bcobachtungen [11] nur gan\% schwache Überschallzustände ausbilden würden, wenn $\lambda=0$ angenommen wird.

Bild 21 zeigt den aus den allgomeinen Stoßgleichungen (17) und (18) borcehneten Druck $\hat{p}$ hinter dern Vordichtungsstoß in Abhängigkeit vorn Gasvolumengehalt der Zuströmung $\alpha_{\infty}$ und cinigen wejteren J'arametern. Aus den dargestellten Frgebnissen erkennt man : $\hat{p}$ steigt mit zunchmendem $\alpha_{\infty}$ und bei festem Zuströmdruck $p_{\infty}$ auch mit wachsender Geschwindigkeit $u_{\infty}$. Sinkt der Druck vor dem StoB nicht auf den Dampfdruck ab, bleibt also $\tilde{p}>p_{v}=0,03$, so ist verständlicherweise a uch $\hat{p}$ entsprechend kleinor als für $\tilde{p}=p_{v}$. Vergleicht man andererseits Strömungsvorgänge, bei denen jeweils vor dem Stoß Dampfdrucl erreicht wird, so ergibt sich : Bei gleichem $\alpha_{\infty}$ und gloicher Kavitationszahl bzw. gleichem Beiwert $\Omega_{w} u_{\infty}^{2} / p_{\infty}$ sinkt die Stoß-Stärke $(\hat{p}-\tilde{p})$ mit abnehmendem $p_{\infty}$.Wert erheblich. Es ist für den Stoß wesentlich, ob bei gleicher Kavitationszahl und gleichem Gasvolumengehalt von hoher oder niedriger Strömungsenergic ausgegangen wird.

Korrigiert man jedoch bei Vorkleinerung des $p_{\infty}$.Wertes den Gasvolumengehalt $\alpha_{\infty}$ entsprechond Gleichung (7) und hält außerdem nicht die Kavitationszahl, sondern die Zuströmgeschwindigkeit konstant, so bleibt die Stoß-Stärko $(\hat{p}-\tilde{p})$ annähernd konstant.

Vergleicht man clic in Bild 21 enthaltenen aus don StoBgleichungen (17), (18) berechneten $\hat{p}$-Werte mit den Drücken $\hat{p}$ dirckt hinter dem Stoß bei den Profilströmungen der Beispiele 1 bis 12 in den $13 i l d e r n ~ 3$ bis 8 und 11 bis 16 , so wird man feststellen, daß letztere $\hat{p}$-Werte etwas kleiner sind, als Bild 21 angibt. 1)er Grund hierfür ist folgendel' :

Boi dom in Ziffer 3 dargelegton Iterationsverfahron zux Berechnung des Profildruchfeldes wird die Stuls. Stärke $\partial(\wp)$ aus Gleichung (35) entsprechend den Stoßrelationen bestimmt. Das Stoßintegral $\oint_{\text {st }}$ in Gleichung (30) hat am Stoß bei $x=x_{1} \mp 0$ die Diskontiniutät $\mp \partial(6) / 2$ mit cinom steilen Abfall vor und hinter dem Stoß. J3ei der in Ziffer 3 orläuterten Einfügung des Stoßintegrals in das Fold 6 geht ein Toil der Diskontinuität dadurch verloren, daß unmittelbar vor dem StoB ein negatives $b$, bzw. ein Wert $p<p_{v}$ entsteht, welcher entsprechend der in Ziffer 3 erläuterten Korrcktur auf $p_{v}$ heraufgesetzt wird. Man könnte natürlich die aus (35) crrechnete Diskontinuität soweit erhöhen, da $\beta$ der $\hat{p}$-Wert aus Bild 21 a uch irn Feld der Profilströmungen an. genommen wird. Die sich dabei ergebenden Druckverläufe hinter dem Stoß wären denjenigen aus den I3ildern 3 bis 8 und 11 bis 16 sehr ähnlich, nur etwas nach oben verschoben.

Wir liaben jodoch aus folgendem Grund von einem solchen Verfahron abgesehen: Einmal wäre delmit der Stoßeinfluß in cinigom Abstand von der Stoßlinie überbewertet. Zum anderen ist aus der schallnahon $\Lambda$ ero. dymamik bekannt, daß die wirklich hinter cinem Vordichtungsstoß auftretenden Drücke stets kleiner sind, als sie durch dio idealisierten Glcichungen für cinen senkrechten Stoß ohne Reibungseinfluß vorhergesagt werden $[10]$.

Es erschien daher sinnvoll, die sich bei dem Rechenverfahren aus Ziffer 3 ergebende Abminderung von $\hat{p}$ gegenüber den Werten aus IBild 21 von etwa 20 bis $25 \%$ nicht zu korrigieren.

Leider stohen sichere Meßwerte übor dic wirklicho Stoß-Stärke in gashaltigem Wasser noch nicht zur Verfügung.

\section{Vergleieh mit lixperimenten}

Für cincn Vorgleich der theoretischen Ergebnisso mit Lxperimenton kömnen aus der uns bekannt gewordenen Jiteratur nur dic Schalldruckmessungen von Srmifas [11] an Tragflügclprofilon in gashaltigem Wasser horan. gezogen werden (vgl. Fußnoto $\left.{ }^{15}\right)$ ). Bezüglich dor Zuströmung und der Profilform sind die Beispiele 9 und 12 etwa mit dem Simmanschen Untersuchungen bei den Anstellwinkeln $\delta_{0}=0^{\circ}$ und $3^{\circ}$ vergleichbar. Die hior theoretisch berechnete Druckverteilung auf der Profilsaugseite mit dem Vordichtungsstoß entspricht ungefähr den Beobachtungen von Srmuax bei den Kavitationsbeiwerten ${ }^{16}$ )

$$
\sigma=\frac{p_{\infty}-p_{v}}{\frac{1}{2} \varrho_{w} u_{\infty}^{2}}=0,73 \text { für } \delta_{0}=0^{\circ} \text { und } \sigma=1,11 \text { für } \delta_{0}=3^{\circ}=0,05 \text {. }
$$

Die Werto der berechnoten Beispicle sind $\sigma=0,56^{\circ}$ für $\delta_{0}=0^{\circ}$ und $\sigma=0,94$ für $\delta_{0}=3^{\circ}$.

Zwar stimmt der Anstieg des Kavitationsbeiwerts mit wachsendem Anstellwinkel zwischen 'l'heoric und lixperiment überoin, und auch die Beiworte $\sigma$ haben etwa die gleiche Größo; für einen wirklichen quantitativen Vergleich fehlen jedoch in der Simнaxschen Untersuchung genaue Angaben über den Gasvolumengehalt $\alpha_{\infty}$ der Zuströmung und die Stärke $\left(\hat{p}-p_{v}\right)$ des Verdichtungsstoßes.

Außerdem ist $\approx$ bedenken, daß in Wirklichkeit keine vollkommen stationäre Strömung auftritt, wio wir sie hier vorausgesetzt haben. Demn einmal sind durch die bereits erwälnte Grenzschichtablösung erhebliche 
instationäre Drucksch wankungen bedingt, die sich auch auf die Lage des Stoßes auswirken. ${ }^{16}$ ) ${ }^{17}$ ) Zun anderen crzwingt der im Verdichtungsstoß3 auftretende Druckanstieg den Zusammenfall der Kavitationsblasen des Ubersohallberoiclis. Hierdurch werden hochfrequente im Kilohertz-Bereich liegende Druekwellen und entsprechende Oszillationen des Verdichtungsstoßes ausgelöst.

Bedenkt man diese hier nur kurz angedeuteten Probleme und dic Schwicrigkeiten ihrer sachgercehten Behandlung, so wird klar, daß dic in der vorliegenden Arbeit entwickelte Theorie nur als ein erster Schritt zur Jerechnung der gashaltigen Wasserströmung um 'Tragflügelprofile angesehen werden kann. Dieses gilt nicht zuletzt auch in Anbetracht der sonstigen strömungsmechanichen und thermodynamischen Vereinfachungen wolche in der vorliegenden Theorie enthalton sind (vgl. Ziffer 1).

\section{Litoratur}

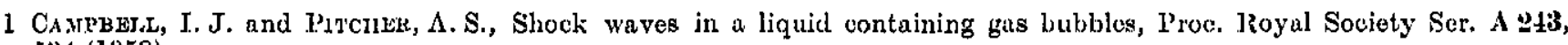
$534(1958)$.

2 WivgharJT, K., lomplessibilitätseffekte in Wasser mit frejem Luftgehalt, Schiffstechnik 14, 24 (1967).

3 Denk, V., Lnisinckil, K. F. und GkotE, H. E., Theoretische und experimentelle Untersuchungen von Gas-Flüssigkeits"Weiphasengemischen, luf tfahrttechnik.Raumfahrttechnik 16, 158 (1970).

4 IsAr, W. H., Zur HIydroak ustik von Propellern in lufthaltigem Wasser, Schiffstechnik 18, 40 (1971).

5 Isay, W. H., Kompressible Strömungen (Ideale Gase, Wasser, lufthaltiges Wasser), Vorlesungammuskript Nr. 5 Instilut für Sohiffbau Hamburg, Februar 1973.

(i ZIEREI, J., Theoric der schallnahen und Hyperschallst römungen, Verlag Hraum, Karlsruhe 1966.

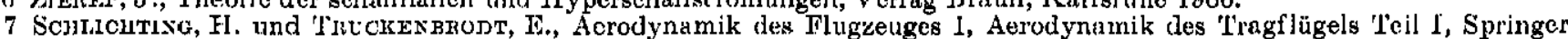
Vorlag, J3erlin-Göttingen.Heidelberg 1959.

8 MEIER, G. and HinLER, W., An experimental investigation of unsteady transsonic flow by high-speed interferometric photography, AGARJ) Confercnce Proc. 'Transsonic Aerodynamics, Sept. 1968.

9 Ronrs, J., Druckverteilungen an symmetrischen Flügelprofilen bei schallnaher Anströmung, Jahrbuch 1959 der WGL, S. 102 .

10 Tyosas, J., Die Ermittlung der Schüttelgrenzen von 'Iragflüggeln im transsonischen Geschwindigkeitsbercich, Jahrbuch 1960 der WGIR, S. 126.

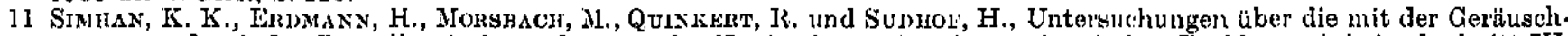
erzeugung durch den Propeller, insbesondere mit der Kavitation verbundenen akustischen Probleme, Arbeitsabsohnitt IV. Bericht des ]3attelle-Instituts Frunkfurt Fobruar 1873.

12 vaN WiJNGaARIJiN, L., On the equations of motion for mixtures of liquid and gas bubbles, Journ. of Wluid Mlecli. 33, 465 (1968);

13 VAx WIJAGARDEN, L., The mechanical behaviour of gas-bubbles in liquids and its effect on the dynamies of liquid-bubblo mixtures, Vortrag GAMIM-Tagung Jelft 1970.

14 Crisio, A., Sound and shock-waves in liquids containing bubbles. The Physies of Fluids 12, 2274 (1969).

15 Starax, K. K., Lin Beitrag zu Strömungsvorgängen in gashaltigen Flïssigkeiten, Diss. Technische Ilochschule Auchen 1970 .

16 Acknirer, J., Feldian, F. und Rotr, N., Lintersuchungen an Verdichtungsstößon in schnell bowegten Gasen, Mitteilungen aus dem Inst. für Aerodynamik der WTH Zürich Nr. I0 (1946).

17 Jizudckxr, G., Jie l3crcchnung der Schültelgrenze von Pfeilflügcln; Zoitschr. f. Flugwiss, 21, 345 (1973),

Dic Mittel für die vorlicgende Untersuchung wurden in dankenswertor Weise durch einen Forschungsauftray im Rahmen des Forsoliungsprogramms der Gruppe Fydroakustik der Fraunhofer-Gescllschaft München-Ottobrunn bereitgestellt. Dje numorischen Rechnungen wurden im Rechenzentrum des Deutschen Elektronensynchrotrons Hamburg dur'chgeführ't. Die Verfassor möchten auch hierfür ihren Dank aussprechen.

Eingereicht am 12.12.1973

Anschrift: l'rof, Dr..Ing. W. H. Isay und Dr. Th. Rowstes, Institut für Schiffbau der Universität Hamburg, Hamburg B0, Lämmerrieth 90, BRD

16) Die angegebenen $\sigma$-Werte entsprechen nach Srunax etwa dem Zustand einer noch gerade anliegenden l'rofilgrenzsehicht hinter dem Stoß. Mit weiter abnehmenden Kavitationsbeiwerten würdo der J)ruckanstieg in Sto B so zunehmen, da B sich die Grenzschicht dahinter ablöst. Der voll abgelöste Zustand ist nach Mlessungen von SransN erreicht bei $\sigma=0,42$ für $\delta_{0,}=0^{\circ}$ und $\sigma=0,48$ für $\delta_{0}=3^{\circ}$.

17) Dieso J) ruckschwankungen sind die Ursache für den aus der transsonischen Aerodynamik bekannten und gefürchteten ,Schüttelvorgang" bei Flugzeugtragf'lügeln [10], [17]. Eine rein theoretische Erfassung dieser Vorgängo stößt noch auf schr' große Schwierigkeiten. 